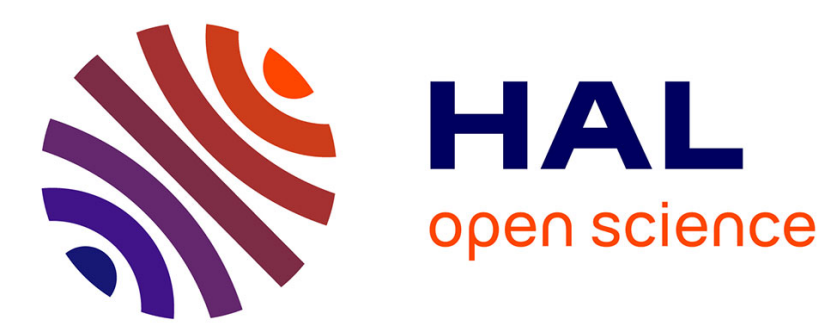

\title{
A Noncooperative Model of Contest Network Formation
}

Kenan Huremovic

\section{To cite this version:}

Kenan Huremovic. A Noncooperative Model of Contest Network Formation. 2016. halshs01154676v2

\author{
HAL Id: halshs-01154676 \\ https://shs.hal.science/halshs-01154676v2
}

Preprint submitted on 23 Sep 2016

HAL is a multi-disciplinary open access archive for the deposit and dissemination of scientific research documents, whether they are published or not. The documents may come from teaching and research institutions in France or abroad, or from public or private research centers.
L'archive ouverte pluridisciplinaire HAL, est destinée au dépôt et à la diffusion de documents scientifiques de niveau recherche, publiés ou non, émanant des établissements d'enseignement et de recherche français ou étrangers, des laboratoires publics ou privés. 


\section{amse}

école d'économie d'aix-marseille

aix-marseille school of economics

\section{Working Papers / Documents de travail}

A Noncooperative Model of Contest Network Formation

Kenan Huremovic 


\title{
A Noncooperative Model of Contest Network Formation*
}

\author{
Kenan Huremović ${ }^{\dagger}$
}

July, 2016

\begin{abstract}
In this paper we study a situation in which agents embedded in a network simultaneously play interrelated bilateral contest games with their neighbors. Spillovers between contests induce complex local and global network effects. We first characterize the equilibrium of the game on a given network. Then we study a network formation model, introducing a novel but intuitive link formation protocol. As links represent negative relationships, link formation is unilateral while link destruction is bilateral. The unique stable network topology is a complete K-partite network with partitions of different sizes. Stable networks exhibit properties that are in line with empirical and theoretical findings from other disciplines.
\end{abstract}

Key Words: network formation, game on network, contest, structural balance JEL: D85, D74

*I am grateful to Fernando Vega-Redondo and Piero Gottardi for their advice. I would also like to thank Stefano Battiston, Yann Bramoulle, Arnaud Dragicevic, Matthias Dahm, Joerg Franke, Timo Hiller, Andrea Mattozzi, Nicola Pavoni, William H. Sandholm, and seminar participants at EUI, SEBS, EEA 2013, 19th CTN Workshop. This paper is based on a chapter of my PhD thesis. I'm grateful to Cooperazione Italiana allo Sviluppo for the financial support. A previous version of this paper was circulated under the name: Rent Seeking and Power Hierarchies: a Noncooperative Model of Network Formation with Antagonistic Links

${ }^{\dagger}$ Aix-Marseille University (Aix-Marseille School of Economics), CNRS, \& EHESS, 2 rue de la Charité, 13236 Marseille cedex 02, France. E-mail: kenan.huremovic@univ-amu.fr, Website: https://sites.google.com/site/kenanhuremovic 


\section{Introduction}

A contest is a strategic interaction in which opposing parties exert costly resources in order to increase their chances to gain control over scarce resources. Contests have been studied in many contexts, including political rent seeking (Hillman and Riley, 1989), discretionary spending of top managers (Inderst et al., 2007), competitions between the departments and schools of universities for funding (Pfeffer and Moore, 1980), sports competitions (Szymanski, 2003), litigation (Sytch and Tatarynowicz, 2014), armed conflict (König et al., 2015). Individuals often compete with several different opponents simultaneously. In this case, the set of bilateral contest relations in a population can be described as a network. We will refer to such a network as a contest network. Contest networks have recently received attention in the literature. For instance, (Sytch and Tatarynowicz, 2014) analyze the network of contest relationships (litigation and antitrust disputes) among US companies and (König et al., 2015) study the network of enmities in the Second Congo War. Figure 1 illustrates a simple network of conflicts among major military forces in Europe from 1872 to 1907.

(a) 1872

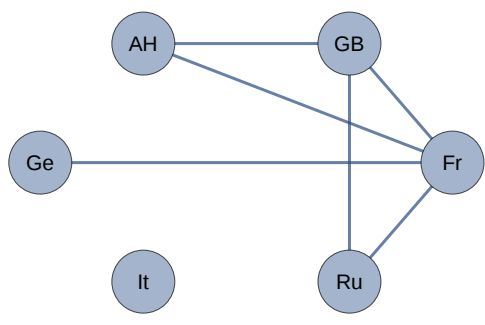

(c) 1904

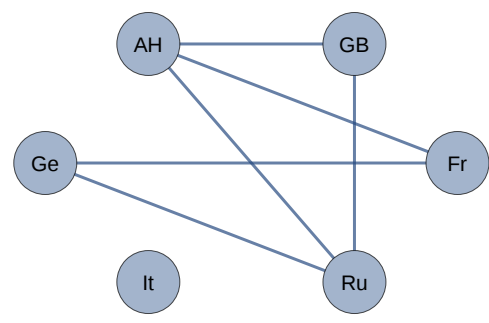

(b) 1890

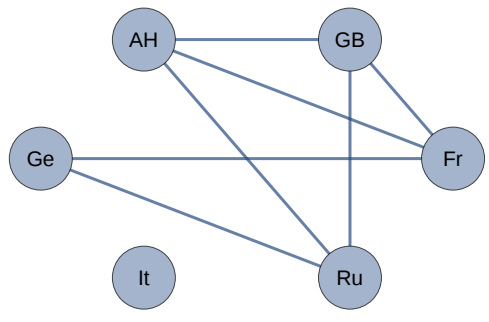

(d) 1907

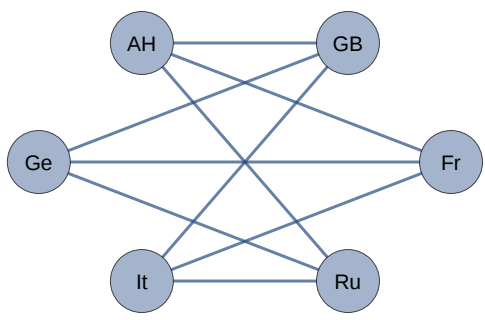

Figure 1: Networks of enmities in Europe (1872 - 1907) (Nodes: Great Britain (GB), France (Fr), Russia (Ru), Italy (It), Germany (Ge), and Austria-Hungary (AH)). Source: Antal et al., 2006)

It has been emphasized in the literature that existing enmity relations affect the further evolution of enmity relations. Examples include interstate conflicts (Levy and Thompson, 2011), and litigation among firms (Sytch and Tatarynowicz, 2014). However, 
a formal treatment of the formation of contest networks has been almost completely neglected in the networks literature ${ }^{1}$.

The main objective in this paper is to study the formation of contest networks and the properties of stable contest networks. To do so, we first analyze the contest game on a given network. When the network is given, agents choose the amount of effort to exert in every specific contest (the effort is link-specific). There are local spillovers across the contests a player is involved in, as she spends the same type of resources (time, money, soldiers) on each of her contests. These spillovers have complex global effects that are mediated by the contest network. Our model of a game on a fixed network can be seen as a modest generalization of the model proposed by (Franke and Ozturk, 2015). Using the same approach as in (Franke and Ozturk, 2015), we show that there is a unique, pure strategy Nash equilibrium of the contest game on a network. Furthermore, we define a concept of a player's strength in the contest network, which is (the strength) endogenous to the model and closely related to the total spending of that player in the equilibrium. The strength of a player is completely determined by the (global) position of that player in the network. Equipped with this result, we turn our attention to the question of network formation.

In the network formation model, agents decide who to engage in contest with (form negative links) and which contests to end (destroy negative links). As the network changes, the effort that players exert in each particular contest will in general change. Thus, our model of network formation can be thought of as a model of network formation coupled with a model of a game on a network. We posit that links can be formed unilaterally (filing a lawsuit, starting a war). On the other hand, to sever a link (ending a litigation process, ending a war), both parties must prefer not to engage in a contest. To the best of our knowledge, this link formation protocol is novel in the literature.

Within this framework, we are primarily interested in understanding which networks are most likely to arise as stable networks. We say that a contest network is stable when no player wishes to create an additional link and no two connected players have an incentive to sever an existing link between them. We allow for simultaneous creation and destruction of links.

The main result of the paper provides a characterization of stable network topologies. We show that in every stable non-empty network, players are partitioned in $K \geq 2$ partitions of unequal sizes. Members of the same partition do not have links with each other, but have links with all other players in the network. So, even though players are ex-ante homogeneous, the stable non-empty network is necessarily asymmetric.

\footnotetext{
${ }^{1}$ Exceptions are (Hiller, 2013) and (Jackson and Nei, 2015). We discuss their contribution later in this paper
} 
The intuition behind the result can be summarized as follows. In the model, a strong player $2^{2}$ has an incentive to form a link with a weak player provided their difference in strength is large enough. This is because it is cheaper to extract resources from weak players than from strong players. As the number of opponents of a weak player increases, she becomes relatively weaker and therefore a more attractive opponent for other strong players.

This mechanism leads to network configurations involving three generic types of players in a stable network. The strongest players in the society (attackers) extract rents from all the players they are linked to. Mixed type players are strong enough to extract resources from the weakest players and at the same time weak enough to be attractive opponents for the strongest players. Weak players are victims. They do not extract resources from anyone, and everyone extracts resources from them. There will always be a single class of attackers and a single class of victims in a stable non-empty network. Remaining $K-2$ classes will be classes of mixed types. There are no links between members of the same class while there is a link between any two players belonging to two different classes.

As transferable contests are wasteful (the effort is costly and the prize is a transfer), an empty network is the unique network topology that maximizes total welfare.

Studying contests has a long tradition in economics, going back to the seminal contributions of (Tullock, 1967) and (Krueger, 1974). The importance of the structure of a contest network has recently been acknowledged in the literature, both theoretically and empirically. Two papers that are close to our work are (Franke and Ozturk, 2015) and (König et al. 2015). The former analyzes a model where individuals are embedded in a network of bilateral contests and choose their degree of fighting effort against their neighbors. The latter studies a conflict model on a network where links can be of two types: enmity links or alliance links. All agents participate in a single $n$-lateral contest and the network structure is built into the payoff function. They also conduct an econometric analysis using data on the Second Congo War, and find that there are significant fighting externalities across contests. However, both (Franke and Ozturk, 2015) and (König et al., 2015 study contests on a given network, while in this paper we are primarily interested in how contest networks are formed and what are the properties of stable contest networks. (Goyal et al., 2016) provide a comprehensive review of the literature on conflict and networks.

To the best of our knowledge, only two other papers study the formation of networks with antagonistic links. In (Hiller, 2013), players form positive links (friendship) and negative links (enmity). A negative link indicates that players are involved in a contest. In

\footnotetext{
${ }^{2}$ Strength is an endogenous concept in our model, and it is a function of the global network structure.
} 
(Hiller, 2013), players do not choose the fighting effort, and a player's strength in a contest is determined by the number of friends she has. In our model, players noncooperatively choose the fighting effort in each of their contests and player's strength depends on the global network structure and on the strengths of all players in the network in a nontrivial way. Furthermore, the link formation protocol and the stability concept employed by (Hiller, 2013) are different from those in our paper. It is interesting, however, that the stable network structures predicted by both models are essentially the same, which indicates the robustness of the results from both papers. (Jackson and Nei, 2015) study the impact of trade on the formation of interstate alliances and on the onset of war. They show that trade can mitigate a conflict.

The rest of the paper is organized in 4 sections. Section 2 lays out the model. In Section 3, we present the main results on stable and efficient networks. Section 4 discusses specific modeling choices and extensions. We conclude in Section 5. All the proofs are given in Appendix A. Appendix B contains an alternative specification of the model.

\section{The Model}

We describe the model starting from the main building block - the bilateral contest game. Then we describe the contest game played on a network and provide results that will be useful to explore network formation. The network formation model is introduced in Subsection 2.3

\subsection{Bilateral contest game}

We model the bilateral contest following (Hillman and Riley, 1989). There are $n=2$ ex-ante identical players competing over a transfer $R$. In this paper we consider players as conflicting parties (countries, tribes, interest groups). The transfer $R$ can be interpreted as loot from armed conflict or damages awarded after litigation.

A player can influence the probability of winning the contest by allocating resources to competing with the opponent. We assume that players simultaneously choose the amount of resources $s_{i} \in[0,+\infty)$ to spend on the contest. The resources are costly, and we denote by $c\left(s_{i}\right)$ the individual cost of spending amount of resources $s_{i}$. We make the following assumptions on the cost function $c$.

\section{Assumption 1}

Function $c: \mathbb{R}_{0}^{+} \rightarrow \mathbb{R}_{0}^{+}$is continuous, twice continuously differentiable with $c(0)=0$, $c^{\prime}(s)>0$ and $c^{\prime \prime}(s)>0$ for all $s \in: \mathbb{R}_{0}^{+}$ 
Let $p_{i}$ be the probability that player $i$ will win the contest. Then the expected payoff of player $i$ who spends the amount $s_{i}$ of resources on the contest with player $j$ is given by:

$$
\pi_{i}\left(s_{i}, s_{j}\right)=p_{i} R-p_{j} R-c\left(s_{i}\right)
$$

Equation (2) defines the outcome probability $p_{i}$.

$$
p_{i}=p_{i}\left(s_{i}, s_{j} ; r\right)=\frac{\phi\left(s_{i}\right)}{\phi\left(s_{j}\right)+\phi\left(s_{i}\right)+r}
$$

A function that maps spendings $s$ into outcome probabilities is known in the literature as a contest success function $(\mathrm{CSF})^{3}$. The parameter $r>0$ determines the probability of a draw (there is no transfer between players in the event of a draw). Here, we assume that $r$ is relatively small. Function $\phi$ is the technology function ${ }^{4}$ which transforms resources allocated to a contest into actual means of fighting. We make the following assumptions about the technology function $\phi$.

\section{Assumption 2}

The technology function $\phi: \mathbb{R}_{0}^{+} \rightarrow \mathbb{R}_{0}^{+}$is:

(i) Continuous and twice differentiable

(ii) Strictly increasing $\left(\phi^{\prime}(s)>0\right)$ and weakly concave $\left(\phi^{\prime \prime} \leq 0\right)$

(iii) $\phi(0)=0$

The first assumption is technical. The second basically imposes non-increasing returns to scale of the technology function. The third assumption guarantees that zero spending implies zero actual means of fighting.

It is worth noting that the game described above has a zero sum flavor, in that the winner of the contest receives a transfer from the loser. Additionally, players spend costly resources on competing. Importantly, this means that when players are homogeneous, the payoff for both players will be negative. So, if there is a choice, players will opt for not playing the contest game.

The bilateral contest game has a unique and symmetric Nash equilibrium in pure strategies (Nti, 1997). When $r$ is small enough, the equilibrium is interior. In this case, the equilibrium strategy of player $i$ is defined with the following implicit function:

$$
\phi^{\prime}\left(s_{i j}^{*}\right) R=\left(r+2 \phi\left(s_{i j}^{*}\right)\right) c^{\prime}\left(s_{i j}^{*}\right)
$$

\footnotetext{
${ }^{3}$ There are several different specifications of CSF considered in the literature, see for example (Jia et al. 2013). The specification used in this paper has been studied in (Nti, 1997)

${ }^{4}$ One can think of it as an analogue of a production function.
} 


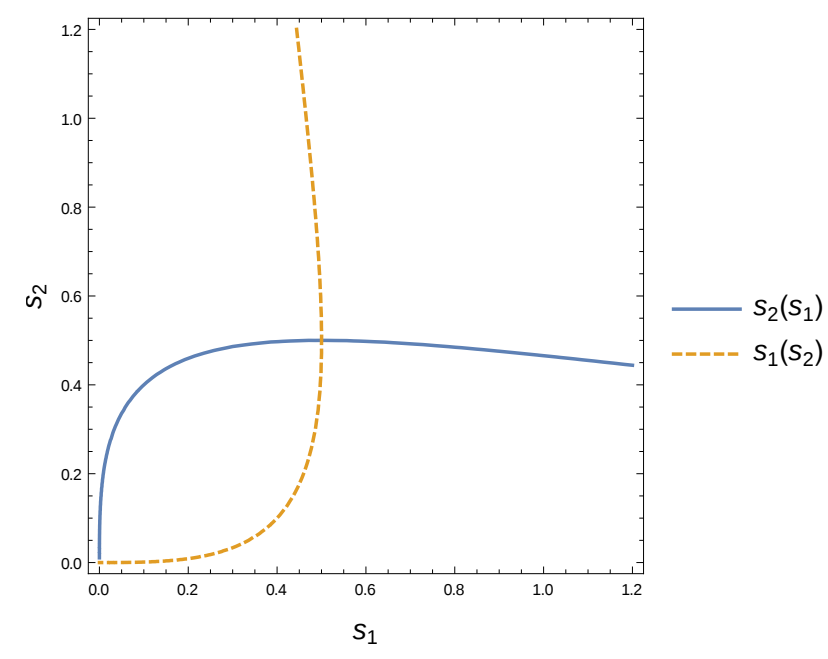

Figure 2: Bilateral contest game - best response functions

The bilateral contest game is neither a game of strategic substitutes nor a game of strategic complements. The best response functions (see Figure 22 are non-linear and nonmonotonic. This property, as we will see in what follows, makes it a real challenge to study the contest game on a network.

\subsection{Contest game on network}

This section is closely related to (Franke and Ozturk, 2015). The difference is that we allow for a positive probability of a draw and a general technology function 5 .

Consider a population composed of a finite set of ex-ante identical agents, $N=$ $\{1,2, \ldots, n\}$. These may be countries or tribes (as in our conflict example), firms, divisions in a multidivisional organization, interest groups. We indicate that $i$ and $j$ play the bilateral contest game by $g_{i j}=g_{j i}=1$ (network $g$ is undirected). The set of contests in the population can be formally described by network $g=\left\{\left(g_{i j}\right)\right\}_{i, j \in N}$. We refer to $g$ as the contest network. Let $N_{i}=\left\{j \in N: g_{i j}=1\right\}$ denote the set of $i$ 's direct neighbors in network $g$. The degree $d_{i}$ of node $i$ is defined by $d_{i}=\left|N_{i}\right|$.

Let $g$ be a contest network. In the contest game on network $g$, every player $i$ chooses the amount of resources $\left(s_{i j}\right)_{j \in N_{i}}$ to devote to each contest she is involved in. Thus, the strategy space of player $i$ is the set $S_{i}=\mathbb{R}_{0}^{+d_{i}}$.

The payoff of player $i$ choosing strategy $\boldsymbol{s}_{i}$ when other agents play $\boldsymbol{s}_{-i}$ is defined by:

$$
\pi_{i}\left(\boldsymbol{s}_{i}, \boldsymbol{s}_{-i} g\right)=\sum_{j \in N_{i}}\left(\frac{\phi\left(s_{i j}\right)}{\phi\left(s_{i j}\right)+\phi\left(s_{j i}\right)+r}-\frac{\phi\left(s_{j i}\right)}{\phi\left(s_{i j}\right)+\phi\left(s_{j i}\right)+r}\right)-c\left(A_{i}\right)
$$

where $A_{i}=\sum_{j \in N_{i}} s_{i j}$ is the total spending of player $i$ in all of her contests. We assume in

\footnotetext{
${ }^{5}$ In $($ Franke and Ozturk, 2015) $\phi$ is assumed to be identity mapping and $r=0$
} 
(3) that the size of the transfer $R$ is independent of the network structure and the same for every contest $\left.g_{i j}\right]^{6}$. We normalize $R=1$ for exposition simplicity ${ }^{7}$,

The specification of the cost function in (3) conveys the idea that there are cost externalities across contests for player $i$, as she spends the same type of resources (money, time) in all her contests. From Assumption 1 it follows that the marginal cost of player $i$ in a contest with $j$ is higher when $i$ spends more in other contests. Therefore, a player's spending in a particular bilateral contest will depend on that player's spending in all her other contests.

We are interested in pure strategy Nash equilibria of the contest game on a network.

\section{Definition 1}

A pure-strategy Nash equilibrium of the contest game on network $g$ is a strategy profile $\boldsymbol{s}^{*} \in S_{1} \times S_{2} \times \ldots \times S_{n}$ such that:

$$
\pi\left(\boldsymbol{s}_{i}^{*}, \boldsymbol{s}_{-i}^{*}, g\right) \geq \pi\left(\boldsymbol{s}_{i}, \boldsymbol{s}_{-i}^{*}\right) \forall\left(\boldsymbol{s}_{i} \in S_{i} \wedge i \in N\right)
$$

The following result will have important implications for our study of network formation.

\section{Proposition 1}

The contest game on a network has a unique pure-strategy Nash equilibrium. There exists $\epsilon>0$ such that the equilibrium is interior when $r<\epsilon$.

Proof. See Appendix A

Proposition 1 is important because it implies that when $r$ is low, the unique purestrategy equilibrium of the contest game will be fully characterized with the set of firstorder conditions of the players' optimization problem. In what follows, we will assume that $r$ is chosen in such a way that the interiority of the equilibrium is guaranteed.

Consider now any two connected players $i$ and $j$. The first-order conditions that characterize their equilibrium behavior in contest $g_{i j}$ are given by:

$$
\left(\frac{\left(r+2 \phi\left(s_{j i}^{*}\right)\right) \phi^{\prime}\left(s_{i j}^{*}\right)}{\left(r+\phi\left(s_{i j}^{*}\right)+\phi\left(s_{j i}^{*}\right)\right)^{2}}-c^{\prime}\left(A_{i}^{*}\right)=0\right) \wedge\left(\frac{\left(r+2 \phi\left(s_{i j}^{*}\right)\right) \phi^{\prime}\left(s_{j i}^{*}\right)}{\left(r+\phi\left(s_{i j}^{*}\right)+\phi\left(s_{j i}^{*}\right)\right)^{2}}-c^{\prime}\left(A_{j}^{*}\right)=0\right)
$$

Simplifying (4) we get the following equation.

$$
\frac{\left(r+2 \phi\left(s_{j i}^{*}\right)\right) \phi^{\prime}\left(s_{i j}^{*}\right)}{\left(r+2 \phi\left(s_{i j}^{*}\right)\right) \phi^{\prime}\left(s_{j i}^{*}\right)}=\frac{c^{\prime}\left(A_{i}^{*}\right)}{c^{\prime}\left(A_{j}^{*}\right)}
$$

\footnotetext{
${ }^{6}$ We use $g_{i j}$ to denote the link between nodes $i$ and $j$ in network $g$ and to refer to the contest between players $i$ and $j$.

${ }^{7}$ This normalization does not have any significant impact on our results
} 
As $\phi^{\prime}(x)>0$ and $\phi^{\prime \prime}(x) \leq 0$ and $c^{\prime \prime}(x)>0$ we have:

$$
A_{i}^{*}>A_{j}^{*} \Rightarrow \frac{c^{\prime}\left(A_{i}^{*}\right)}{c^{\prime}\left(A_{j}^{*}\right)}>1 \Rightarrow \frac{\left(r+2 \phi\left(s_{j i}^{*}\right)\right) \phi^{\prime}\left(s_{i j}^{*}\right)}{\left(r+2 \phi\left(s_{i j}^{*}\right)\right) \phi^{\prime}\left(s_{j i}^{*}\right)}>1 \Rightarrow s_{j i}^{*}>s_{i j}^{*}
$$

where the last implication in (5) is due to the facts that $\phi$ is an increasing function and $\phi^{\prime}$ is a decreasing function.

In the equilibrium the player whose total spending is lower will win a contest with higher probability. This observation reflects the fact that the more 'exhausted' player (the one who spends more resources in the equilibrium) performs worse in a particular contest. The reason is that an additional unit of resources is more costly for her due to the convexity of the cost function. This apparently counterintuitive observation - that a player spending more in total loses 8 a contest against player spending less in total - will be used extensively in the next section. Based on the above considerations, we introduce our notion of relative strength of players.

\section{Definition 2}

Player $i \in N$ is said to be stronger than player $j \in N$ in contest network $g$ if $A_{i}^{*}<A_{j}^{*}$

Note also that the equilibrium strategy of a player is uniquely determined by her position in the contest network. This is because the network $g$ essentially determines the system of first-order condition equations. As spillovers across contests are global, it is not just the local structure of the network that matters. However, finding an explicit relation between the position of a player in the network and her equilibrium strategy proved to be infeasible. In Section 5 we briefly discuss this issue.

\subsection{Network formation}

We now explore which contest networks are likely to arise. To understand which networks are strategically stable networks, we first need to define how links can be formed and destroyed (link formation protocol). In this paper, a link between two players indicates that they are engaged in an antagonistic interaction. To start such an interaction, action by one party suffices. For instance, to start a war one country waging war is sufficient; or one side filing a lawsuit is sufficient to start a litigation process. On the other hand, for there to be no contest, both parties have to agree. Thus, in our model of network formation, links are formed unilaterally while the destruction of a link is a bilateral decision. When the interaction is such that the existence of a link implies an adversary relationship, we believe the above link formation protocol is the natural one. To the best of our knowledge, this is the first paper in the literature to consider such a link formation protocol.

\footnotetext{
${ }^{8}$ We say that agent $i$ loses the contest against player $j$ when $p_{i}^{*}<p_{j}^{*}$
} 
Informally, we say that network $g$ is stable when (a) no player wants to create additional links, and (b) no player can improve her payoff by creating a set of links while simultaneously severing some existing links, and (c) no player can improve her payoff by severing links.

Let us now formally define our concept of stability. First, we introduce the notion of action equilibrium, and then define the concept of stability we use.

Definition 3 (Action equilibrium)

A network $g$ is in action equilibrium if all actions $s_{i j}$ and $s_{j i}$ assigned to every contest $g_{i j} \in g$ are part of the unique Nash equilibrium of the game on a fixed network

Definition 4 (Stable network)

A network $g$ in action equilibrium is said to be a stable network if (6) holds for every agent $i \in N$ and any two (possibly empty) sets of nodes $A \subseteq N$ and $B \subseteq N$

(i) $\quad \pi_{i}\left(g+\left\{g_{i j}\right\}_{j \in A}\right) \leq \pi_{i}(g)$

(ii) $\quad \pi_{i}\left(g+\left\{g_{i j}\right\}_{j \in A}-\left\{g_{i j}\right\}_{j \in B}\right)>\pi_{i}(g) \Rightarrow(\exists j \in B): \pi_{j}\left(g-g_{i j}\right)<\pi_{j}(g)$

Definition 4 implies that players are allowed to remove and replace multiple links at the same time. Allowing players to manipulate several links at the same time is not crucial for our results - as can be seen from the proofs of our claims. Assuming that players can create, sever or replace only one link at a time would not affect the results of the paper. On the other hand, the assumption that players can replace a link is necessary for our results. This assumption is made in other models of network formation, including (Watts, 2001) and (Goyal and Vega-Redondo, 2007).

When deciding on her links, player $i$ knows the network $g$ and the equilibrium strategy profile $\boldsymbol{s}^{*}$. A player will create a link only if it is strictly profitable for her to do so. If a player is indifferent between keeping or destroying a link, the link will be destroyed. Players, therefore, prefer to be involved in a smaller number of contests. This tie-breaking rule does not have any significant effect on our results.

In our model of network formation, agents are myopic. As our model combines both network formation and playing a game on a network, the assumption of myopic behavior in the model has twofold meaning.

Assumption 3 (i) A player decides to add or sever links under the assumption that players in the network will play the same strategy in all other remaining contests. The effort levels $\left(s_{i j}, s_{j i}\right)$ assigned to the newly-formed link $g_{i j}$ are determined as the solution of the bilateral contest game, keeping all other actions in the network fixed (see Example 1) 
(ii) Agents do not take into account future changes of network $g$ that may be the result of their adding or severing links

We believe that (i) in Assumption 3, apart from appearing in the literature9 and being convenient, is natural. Solving for the Nash equilibrium of the game on a fixed network is analytically intractable, and computationally intensive. The reason is that the equilibrium is characterized by the first-order conditions (4), which is a system of $\left|\left\{g_{i j}\right\}_{i, j=1}^{n}\right|$ non-linear equations. So it may be unsound to assume that players have the cognitive ability to predict all the adjustments that will take place in all the contests in the network when they create or sever a link. Furthermore, we show in Subsection 4.1 that strategy profile $s$ of the game on a fixed network will globally converge to the action equilibrium when agents follow a simple and natural myopic strategy updating process. We use this result in Subsection 4.1 to justify the notion of stability from Definition 4 as a steady state of a coupled dynamic process of network formation and playing the game on a network. Item (ii) in Assumption 3 is prevalent in the network formation literature (Jackson, 2008), and does not require further elaboration.

\section{Example 1}

Let $c(x)=\frac{1}{2} x^{2}$. Suppose network $g$ is in action equilibrium, and denote with $A_{i}^{*}, A_{j}^{*}$ the total equilibrium spending of players $i$ and $j$ respectively. When link $g_{i j}$ is created, corresponding actions $s_{i j}$ and $s_{j i}$ are determined as the solution of:

$$
\begin{aligned}
& \frac{2 s_{j i}-r}{\left(s_{i j}+s_{j i}+r\right)^{2}}=\left(A_{i}^{*}+s_{i j}\right) \\
& \frac{2 s_{i j}-r}{\left(s_{i j}+s_{j i}+r\right)^{2}}=\left(A_{j}^{*}+s_{j i}\right)
\end{aligned}
$$

which is given by

$$
s_{i j}=\frac{2+A_{i}^{\prime}\left(A_{i}^{\prime}+A_{j}^{\prime}-\sqrt{4+\left(A_{i}^{\prime}+A_{j}^{\prime}\right)^{2}}\right)}{2 \sqrt{4+\left(A_{i}^{\prime}+A_{j}^{\prime}\right)^{2}}}>0
$$

Here $A_{i}^{\prime}=A_{i}^{*}-r / 2$. Player $i$ will wish to form link $g_{i j}$ when

$$
\frac{s_{i j}-s_{j i}}{\left(s_{i j}+s_{j i}+r\right)}+A_{i}^{* 2}-\left(A_{i}^{*}+s_{i j}\right)^{2}>0
$$

and $\left(s_{i j}, s_{j i}\right)$ are determined with (7), and analogously for player $j$.

On the other hand, the existing link $g_{i j}$ will be destroyed if both players agree to destroy it, that is when $\pi_{i}\left(\mathbf{s}_{i}, \mathbf{s}_{-i}, G-g_{i j}\right) \geq \pi_{i}\left(\mathbf{s}_{i}, \mathbf{s}_{-i}, G\right)$ and $\pi_{j}\left(\boldsymbol{s}_{j}, \boldsymbol{s}_{-j}, g-g_{i j}\right) \geq \pi_{i}\left(\boldsymbol{s}_{j}, \boldsymbol{s}_{-j}, g\right)$. This will be the case when

$$
A_{i}^{* 2}-\left(A_{i}^{*}-s_{i j}^{*}\right)^{2}-\frac{s_{i j}^{*}-s_{j i}^{*}}{\left(s_{i j}^{*}+s_{j i}^{*}+r\right)} \geq 0 \wedge A_{j}^{* 2}-\left(A_{j}^{*}-s_{j i}^{*}\right)^{2}-\frac{s_{j i}^{*}-s_{i j}^{*}}{\left(s_{i j}^{*}+s_{j i}^{*}+r\right)} \geq 0
$$

\footnotetext{
${ }^{9}$ See for example (Jackson and Watts, 2002 ) who make the same assumption
} 


\section{Analysis}

Consider network $g$ which is in the action equilibrium. We can rank all players in the network in increasing order with respect to their total equilibrium spending $\left(A_{1}^{*}<A_{2}^{*}<\right.$ $\ldots<A_{K}^{*}$ ), where $K \leq n$ is the number of different total spending levels in the network. We denote by $\mathscr{A}_{i}$ the class of nodes whose total equilibrium spending is $A_{i}^{*}$.

We say that player $i$ has control over link $g_{i j}$ if it is beneficial ${ }^{10}$ for player $j$ to destroy link $g_{i j}$. Thus, when agent $i$ has control over $g_{i j}$ it is completely up to her whether the link is destroyed. This is an important notion as the link destruction is a bilateral action. Note that both $i$ and $j$ are in control of link $g_{i j}$ when this link is not beneficial to either of them. Recall that by Definition 2 we say that player $j$ is stronger than player $i$ when $A_{i}^{*}>A_{j}^{*}$. In general, we refer to $A_{i}^{*}$ as the strength of player $i$ (higher $A_{i}^{*}$ implies a weaker player $i$ ). It is clear that when $i$ is stronger than $j$, then $i$ controls link $g_{i j}$.

\section{Example 2}

Consider network $g$ depicted in Figure 3 .

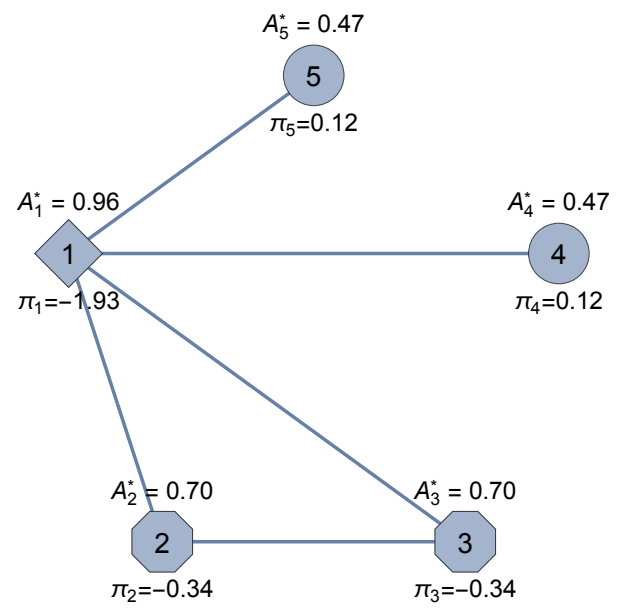

Figure 3: Contest network

It is important to note that the above concept of strength is endogenous and crucially depends on the global structure of the network. When the contest network changes, then in general all strengths of all players in the network will change.

The total spending of each node in the action equilibrium (strength) is indicated above the node. There are three classes of node in network $g$, based on their total equilibrium spending - members of the same class have the same vertex shape. Agents 4 and 5 are the strongest agents, while agent 1 is the weakest. Agent 4 is in control of link $g_{41}$, since

\footnotetext{
${ }^{10} \mathrm{~A}$ link $g_{i j}$ is said to be beneficial for player $i$ if the creation of this link (if it did not exist) makes player $i$ better off and if the destruction of this link (if it exists) makes player $i$ worse off
} 
agent 1 would prefer to sever link $g_{41}$, as this would increase her payoff. Agent 1 is not in control of link $g_{41}$, as agent 4 prefers to keep this link. Both agents 3 and 2 are in control of link $g_{32}$, as both of them would like to sever it. The network in Figure 3 is obviously not stable.

Let us define the net payoff of player $i$ from contest $g_{i j}$ with:

$$
\pi_{i}\left(g_{i j}\right)=\frac{\phi\left(s_{i j}^{*}\right)-\phi\left(s_{j i}^{*}\right)}{\left(\phi\left(s_{i j}^{*}\right)+\phi\left(s_{j i}^{*}\right)+r\right)}-\left(c\left(A_{i}^{*}\right)-c\left(A_{i}^{*}-s_{i j}^{*}\right)\right)
$$

We now state the result which enables us to compare different contests of a player, and which will be very useful in studying stable contest networks.

\section{Proposition 2}

Let $a \in \mathscr{A}_{i}, b \in \mathscr{A}_{j}, c \in \mathscr{A}_{k}$ such that $i<j<k$ and $g_{a b}, g_{a c} \in g$. Then $s_{a b}^{*}>s_{a c}^{*}$, $s_{b a}^{*}>s_{c a}^{*}$, furthermore $\pi_{i}\left(g_{a b}\right)<\pi_{i}\left(g_{a c}\right)$

Proof. See Appendix A

Proposition 2 contains an important intuition for our main result. It states that a strong player contesting two players $j$ and $k$ spends less and has a higher expected payoff in the contest with the weaker player of the two. Thus, a strong player has an incentive to compete with the weakest agents in the society.

Our main result states that a stable network has to be a complete K-partite network with partitions of different sizes. We prove this result by proving the set of intermediate results, reducing the set of possible stable networks. Let us first introduce the following definition.

\section{Definition 5}

Player $a \in \mathscr{A}_{i}$ is an attacker if all of her contests are with agents from $\overline{\mathscr{A}}_{i}=\left\{\mathscr{A}_{j} \mid j>i\right\}$. Player $a \in \mathscr{A}_{i}$ is a mixed type if there exist players $b$ and $c$ such that $g_{a b}, g_{a c} \in g$ and $A_{b}^{*}>A_{a}^{*}>A_{c}^{*}$ Player $a \in \mathscr{A}_{i}$ is a victim if she has all of her contests are with agents from $\underline{A}_{i}=\left\{\mathscr{A}_{j} \mid j<i\right\}$

It is clear that every player $i$ is one of 3 types defined in Definition 5. Note also that, in a stable network, all attackers must receive a positive payoff from all their contests. If this is not true for some attacker $i$, then since she controls all her links, she could deviate and destroy non-profitable links. Furthermore, any player $j$ in a stable network must be stronger than all opponents of some attacker $i$ in order not to be connected to $i$. This is because an attacker always has an incentive to switch from a strong to a weak opponent. We state this formally in Lemma 1 . 


\section{Lemma 1}

Let $a \in \mathscr{A}$, and $\mathscr{A}$ is a class of attackers. Let $b$ and $c$ be two nodes in the network such that $A_{b}^{*} \leq A_{c}^{*}, g_{a b}=1$ and $g_{a c}=0$. Replacing $g_{a b}$ with $g_{a c}$ is a profitable deviation for player $a$.

Proof. See Appendix A

Figure 4 illustrates the statement of Lemma 1.

(a)

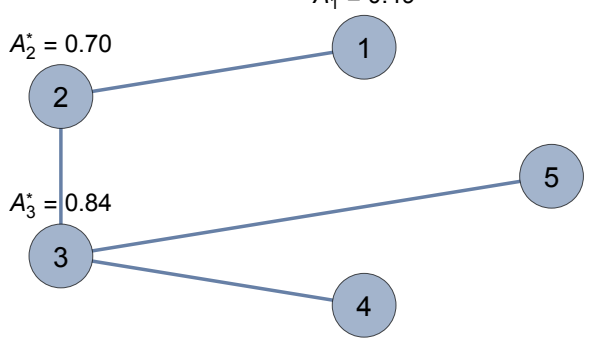

(b)

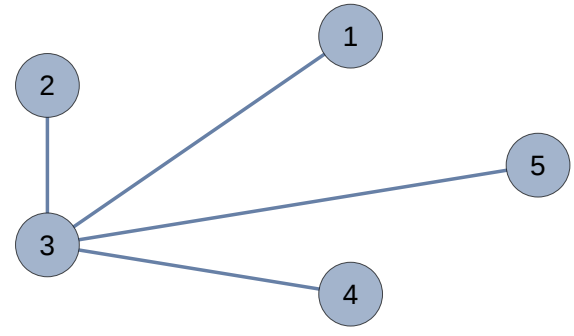

Figure 4: Profitable deviation - player 1 replaces $g_{12}$ with $g_{13}$, network (a) becomes network (b)

The following corollary is direct consequence of Lemma 1.

\section{Corollary 1}

If in a stable network player $a \in \mathscr{A}_{i}$ has a link with player $b \in \mathscr{A}_{j}$ and $i<j$ then she has a link with every player $c \in \mathscr{A}_{j+k} k=0,1,2, . . K-j$

Proof. See Appendix A

We next show that a non-empty stable network must be connected. Recall that Lemma 1 states that if there exists a player $k$ in the network such that $A_{k}^{*}<A_{j}^{*} \wedge g_{i k}=$ $0 \wedge g_{i j}=1$ and player $i$ has control over $g_{i j}$, she will have an incentive to replace $g_{i j}$ with $g_{i k}$. We argue that this implies that there cannot be more than one component in a stable non-empty network. To see this, suppose that, contrary to what is asserted, a stable network is split into two or more components. Consider two components such that attackers in those components earn a positive payof ${ }^{11}$. Let $m$ be the weakest player in these two components. Then, by Lemma 1, an attacker from the component that does not include $m$ has an incentive to sever one of her links and form a link with $m$. We state this result in Lemma 2

\footnotetext{
${ }^{11}$ We present the arguments more formally in the proof in the appendix
} 


\section{Lemma 2}

A non-empty stable network must be connected

Proof. See Appendix A

Example 3 illustrates the intuition behind Lemma 2

\section{Example 3}

In (a) node 3 who is an attacker has an incentive to form a link with the weakest player in the network, which results in network (b). In (b), both player 2 and player 1 have incentive to sever link $g_{12}$ and form a link with player 4.
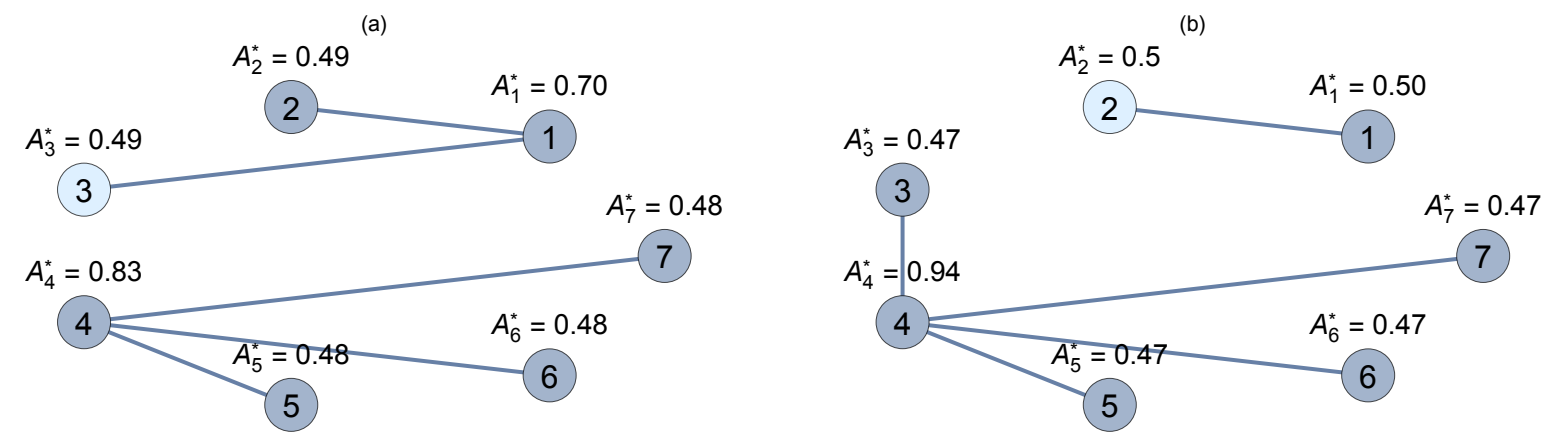

Figure 5: Profitable deviation in case of not-connected network

In the rest of the paper we will take it as given that the stable network is connected.

Our results so far are based on the fact that weak players are attractive opponents. Let us now focus on attackers. By definition, an attacker has control over all her links. Therefore, it is always feasible for an attacker to imitate the linking strategy of another attacker. Building on this observation, we can state the following lemma.

\section{Lemma 3}

Two players that belong to the same class of attackers $\mathscr{A}$ have the same neighborhood

Proof. See Appendix A

Since all attackers in the same class have the same neighborhood in a stable network, it must be that they have the same payoff. We argue now that there can be at most one class of attackers. Suppose that there is more than one class of attackers and that members of different classes of attackers have different payoffs. Since attackers have control over their links, we can show that members of a class with low payoff have an incentive to imitate the strategy of members of a class with high payoff. And since they are attackers, this deviation is feasible under the link formation protocol. This implies that all attackers in 
a stable network must have the same payoff. Furthermore, the incentive to attack weaker players will incite all attackers to have the same neighborhood. Eventually, the network will have only one class of attackers. We formalize this intuition in the following lemma.

\section{Lemma 4}

There is only one class of attackers in a stable network

Proof. See Appendix A

Lemma 4 and Corollary 1 imply that members of the unique class of attackers are connected to all nodes in the network that are not attackers. To see this, note that class $\mathscr{A}_{2}$ must be a class of mixed types or victims, as there is only one class of attackers. In either case, Lemma 4, together with the fact that two players from the same class cannot be connected in a stable network, implies that all members of $\mathscr{A}_{1}$ and $\mathscr{A}_{2}$ are connected. Then Corollary 1 implies that attackers are connected to all other nodes in the network.

Let us now turn to mixed types in a stable network. Using the same reasoning as in the case of attackers, we can conclude that all members of a mixed class must have the same neighbors in the subset of players that are weaker than they are. We show that the same will hold with respect to their stronger neighbors. We state this result as a separate lemma.

\section{Lemma 5}

In a stable network all members of a mixed type class are connected to all other nodes in the network that do not belong to their class.

Proof. See Appendix A

Lemma 3 and 5 directly imply:

\section{Corollary 2}

There is only one class of victims and all victims have the same neighborhood

Proof. Omitted

We are now ready to state the main result of the paper regarding the structure of stable networks.

\section{Proposition 3}

A stable network is a complete $K$-partite network

Proof. See Appendix A 
An example of a stable network is given in Figure 6

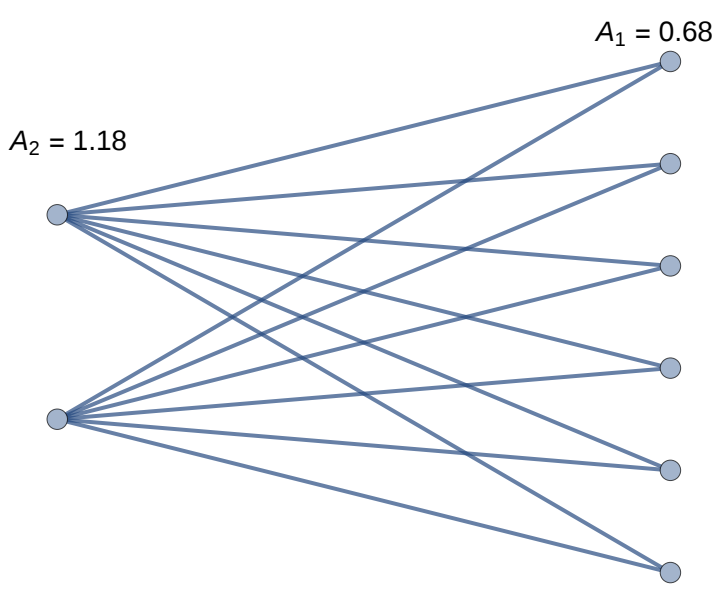

Figure 6: A stable network - complete K-partite, $K=2$

We have shown that in a stable network we can partition all agents into $K<n$ different classes with respect to their equilibrium spending. Members of the same class are in contest with all agents who are not members of their own class. The classes in a stable network cannot be of the same size. If this were the case, all players in the network would have the same total spending and therefore no contest would be profitable for any of the players. In Proposition 4, we note that stronger classes (classes with low total equilibrium spending per node) must have more members than weaker classes.

\section{Proposition 4}

Let $\left|\mathscr{A}_{k}\right|$ denote the number of nodes that belong to class $\mathscr{A}_{k}$. Then $\left|\mathscr{A}_{k}\right|>\left|\mathscr{A}_{k+1}\right| \forall k \in$ $\{1, \ldots, K-1\}$

\section{Proof. See Appendix A}

It is clear that $\left|\mathscr{A}_{k}\right|>\left|\mathscr{A}_{k+1}\right|$ is not a sufficient condition for the stability of the network. The difference between $\left|\mathscr{A}_{k}\right|$ and $\left|\mathscr{A}_{k+1}\right|$ must be large enough to ensure that members of the stronger class do not find it payoff-improving to sever links with members of the weaker class. Links that can be created in a complete K-partite network are links between players from the same partition and therefore with the same strength. Note, however, that in a complete K-partite network, no player will wish to create a link,since, as we have seen before, link $g_{i j}$ such that $A_{i}^{*}=A_{j}^{*}=A$ cannot be profitable.

Let us now discuss the sufficient conditions for the stability of a complete K-partite network. For simplicity let us assume that $r \rightarrow 0$, and that $\phi$ is the identity mapping. As discussed above, a sufficient condition for stability is that no player wants to sever a link. 
No player will want to destroy a link if every contest is profitable for one of the players. We can express the sufficient conditions for stability of a network in terms of the total spending in the equilibrium.

\section{Proposition 5}

A complete K-partite network will be stable if for every contest $g_{i j}$

$$
\frac{c^{\prime}\left(A_{j}^{*}\right)-c^{\prime}\left(A_{i}^{*}\right)}{c^{\prime}\left(A_{j}^{*}\right)+c^{\prime}\left(A_{i}^{*}\right)}>c\left(A_{i}^{*}\right)-c\left(A_{i}^{*}-\frac{2 c^{\prime}\left(A_{j}^{*}\right)}{c^{\prime}\left(A_{i}^{*}\right)+c^{\prime}\left(A_{j}^{*}\right)}-\frac{r}{2}\right)
$$

Proof. See appendix A

We now focus on a particular class of complete $K$-partite networks - bipartite networks $(K=2)$. Assume, for simplicity, that the cost function is given by $c(x)=x^{2}$. Denote the two partitions by $\mathscr{K}_{1}$ and $\mathscr{K}_{2}$, and the sizes of the partitions by $k_{1}$ and $k_{2}$ respectively. Let $k_{1}>k_{2}$. Due to the symmetry, agents that belong to the same partition will play the same strategy in every contest $g_{i j}$. Then, the total effort of members of the two partitions can be written as $A_{1}^{*}=k_{2} s_{1}^{*}$ and $A_{2}^{*}=k_{1} s_{2}^{*}$, where $s_{i}^{*}, i \in\{1,2\}$ is the effort level in each contest of members of partition $\mathscr{K}_{i}$. The following proposition holds.

\section{Proposition 6}

A complete bipartite network with sizes of partition $k_{1}$ and $k_{2}\left(k_{1}>k_{2}\right)$ will be stable when $k_{1}>\frac{1-4 k_{2}+6 k_{2}^{2}+\left(2 k_{2}-1\right) \sqrt{1-4 k_{2}+8 k_{2}^{2}}}{2 k_{2}}$

Proof. See Appendix A

The payoff of a player $i \in \mathscr{K}_{1}$ increases with $k_{1}$, and is non-monotone in $k_{2}$. Specifically, the payoff of player $i \in \mathscr{K}_{1}$ will increase in $k_{2}$ as long as: $2\left(k_{1}^{\frac{3}{2}}-k_{2}^{\frac{3}{2}}\right)-k_{1}^{\frac{1}{2}} k_{2}^{\frac{1}{2}}\left(3 k_{1}^{\frac{1}{2}}+\right.$ $\left.5 k_{2}^{\frac{1}{2}}\right)>0$ The payoff of a member of the larger partition is affected in two ways when the size of the smaller partition increases. First, contests become more costly, as the members of the smaller partition become stronger. The second effect is that players from $\mathscr{K}_{1}$ have more opponents to extract resources from. Depending on which effect dominates, the payoff of agents from the larger partition will increase or decease with the size of the smaller partition. 


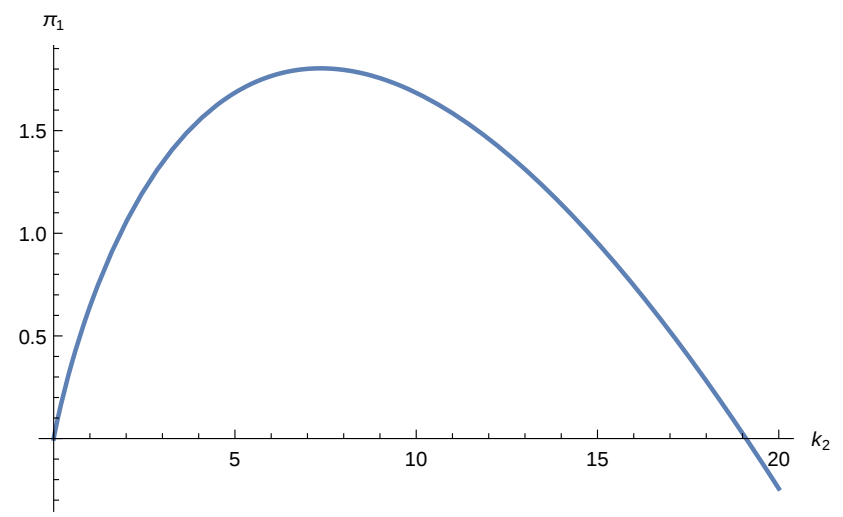

Figure 7: Payoff of members of partition $\mathscr{K}_{1}$ as a function of $k_{2} . k_{1}=50$

\subsection{Efficiency}

It is easy to show that the unique network structure that maximizes the total utility of society is an empty network. This is a direct consequence of the transferable nature of the contest game and the fact that effort is costly. Indeed, the total payoff that society obtains from network $g$ can be expressed as:

$$
\begin{aligned}
U(g) & =\sum_{i \in N} \pi_{i}\left(\mathbf{s}_{i}, \mathbf{s}_{-i} ; g\right) \\
& =\sum_{i \in N} \sum_{j \in N_{i}}\left(\frac{\phi\left(s_{i j}\right)}{\phi\left(s_{i j}\right)+\phi\left(s_{j i}\right)+r}-\frac{\phi\left(s_{j i}\right)}{\phi\left(s_{i j}\right)+\phi\left(s_{j i}\right)+r}-c\left(A_{i}\right)\right) \\
& =-\sum_{i \in N} c\left(A_{i}\right)
\end{aligned}
$$

The following proposition directly follows from the above discussion

\section{Proposition 7}

The efficient network is the empty network

Proof. Omitted

\section{Discussion}

\subsection{Dynamics of network formation}

We can think of the network formation process in this paper as a discrete dynamic process. Time is indexed with $t \in \mathbb{N} \cup\{0\}$. In period $t=0$ an arbitrary contest network $g$ is given 12 . For every period $t$ :

\footnotetext{
${ }^{12}$ Due to the 'negative sum' nature of the contest game, the empty network will always be stable in our model. In order to describe the dynamic process that leads to a non-empty stable network, we
} 
(i) At the beginning of period $t$ the network from $t-1$ is in the action equilibrium

(ii) Random player $i$ is chosen and she updates her links according to the link formation protocol, resulting in network $g_{t+1}$ (which is not in the action equilibrium)

(iii) The second dynamic process (action adjustment process ${ }^{13}$ ) starts, and all agents update their strategies in contests on network $g_{t+1}$ according to the process formally described in what follows.

Example 4 illustrates the formation process

\section{Example 4}

Let us consider a path from network (a) to stable network (d) in Figure 8.

(a)

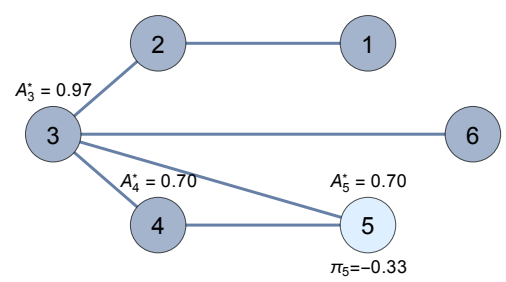

(c)

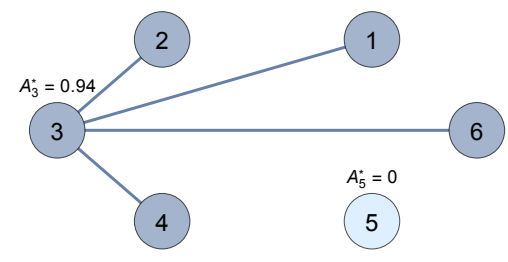

(b)

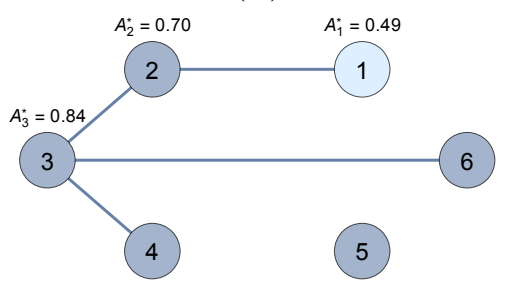

(d)

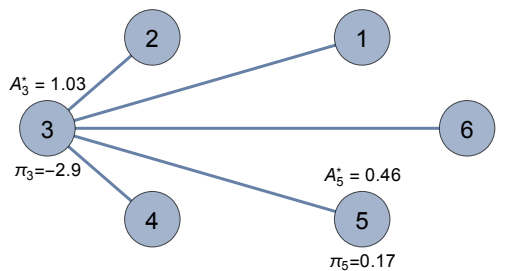

Figure 8: An illustration of network formation process

$(\boldsymbol{a}) \rightarrow(\boldsymbol{b})$ : In network (a) node 5 is chosen to update her linking strategy. Link $g_{54}$ is not profitable for 5, as 4 and 5 have the same strength. Link $g_{53}$ is not profitable for 5, even though 5 is stronger than 3. Thus, it is profitable for player 5 to update her linking strategy by severing links $g_{54}$ and $g_{53}$. This action is feasible, as 5 has control over her links.

$(\boldsymbol{b}) \rightarrow(\boldsymbol{c}):$ Player 1 updates her strategy. She finds it profitable to replace link $g_{12}$ with

assume that, because of some non-modeled mutation or a tremble, the initial conditions are given with a potentially non-empty network.

${ }^{13}$ We will assume that this process takes place in continuous time (so it happens on a much faster time-scale than the network formation process, which is discrete). 
link $g_{13}$, as 3 is weaker than 2. This is a profitable deviation according to Lemma 1 $(\boldsymbol{c}) \rightarrow(\boldsymbol{d})$ : Only node 5 in network (c) has an incentive to update her linking strategy. No other node in (c) has an incentive to change her strategy (3 would prefer to destroy her links, but cannot because links incident to 3 are beneficial to opponents of 3). 5 forms link $g_{53}$, which is profitable for her. The network in (d) is a stable network (complete bipartite).

Let us formally describe the action adjustment process. We assume that player $i$ updates her strategy following a simple gradient-based adjustment as defined in equation (9)

$$
\frac{d \mathbf{s}_{i}}{d t}=\alpha \nabla_{i} \pi_{i}(\mathbf{s}), \alpha>0, i=1, . ., n
$$

where $\pi_{i}(\mathbf{s})=\pi_{i}\left(\mathbf{s}_{1}, \mathbf{s}_{2}, \ldots, \mathbf{s}_{i}, \ldots \mathbf{s}_{n}\right)$ and $\nabla_{i} \pi_{i}(\mathbf{s})=\left(\begin{array}{cccc}\frac{\partial \pi_{i}}{\partial s_{i 1}} & \frac{\partial \pi_{i}}{\partial s_{i 1}} & \ldots & \frac{\partial \pi_{i}}{\partial s_{i d_{i}}}\end{array}\right)$ is the gradient of the payoff function with respect to $\boldsymbol{s}_{\boldsymbol{i}}$. It is clear that the pure-strategy Nash equilibrium defined in Definition 1 is a steady state of this dynamics. We prove that this Nash equilibrium is a globally asymptotically stable state of (9). Let us define function $J$ : $\prod_{i}[0, M]^{d_{i}} \rightarrow \prod_{i}[0, M]^{d_{i}}$ with:

$$
J(\mathbf{s})=\left(\begin{array}{c}
\nabla_{1} \pi_{1}(\mathbf{s}) \\
\nabla_{2} \pi_{2}(\mathbf{s}) \\
\ldots \\
\nabla_{n} \pi_{n}(\mathbf{s})
\end{array}\right)
$$

We can now write system $(9)$ in a more compact form

$$
\dot{\mathbf{s}}=\alpha J(\mathbf{s})
$$

To prove global stability, we need to show that the rate of change of $\|J\|=J J^{\prime}$ is always negative (and equal to 0 in the equilibrium). Let us denote by $G$ the Jacobian matrix of $J$. The following holds.

$$
\frac{d}{d t} J J^{\prime}=(G \dot{\mathbf{s}})^{\prime} J+J^{\prime} G \dot{\mathbf{s}}=\left(J^{\prime} G^{\prime} J+J^{\prime} G J\right)=J^{\prime}\left(G^{\prime}+G\right) J
$$

From the proof of Proposition 1, it directly follows that $\left(G^{\prime}+G\right)$ is a negative definite matrix. This implies that $\frac{d}{d t} J J^{\prime}<0$, which is what we had to prove. Thus, if every player adjusts her actions according to the adjustment process in (10), the action adjustment process converges, irrespective of the initial conditions. The discussion above implies the following proposition.

\section{Proposition 8}

The action adjustment process given by equation 10 is globally asymptotically stable 
Proof. See Appendix A

It is interesting to note that Proposition 8 has a very practical application. It provides an efficient way to numerically calculate the action equilibrium on an arbitrary network.

It is clear that the described network formation process is a path dependent process. Example 5 illustrates how starting from the same initial condition (a) the network formation process can lead to two different stable networks $(d)$.

\section{Example 5}

Figure 9 illustrates path dependency of the network formation process. The resulting stable network depends on the order in which agents are chosen to update their linking strategy. The agent which updates linking strategy is indicated with light blue color of the node

(a)

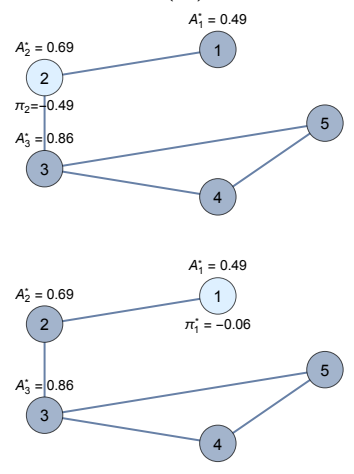

(b)
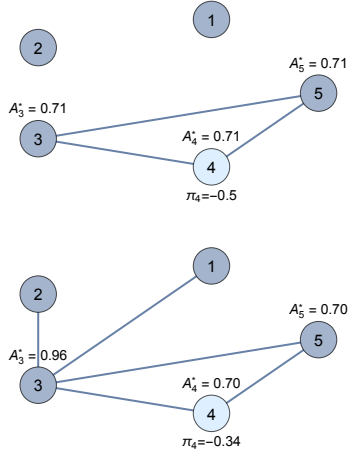

(c)

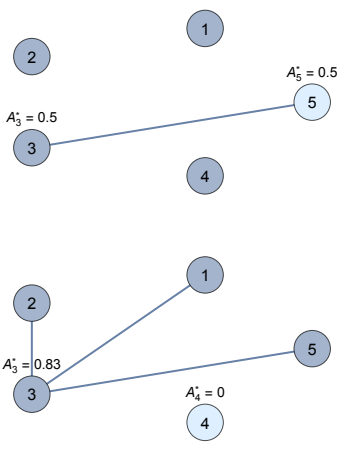

(d)

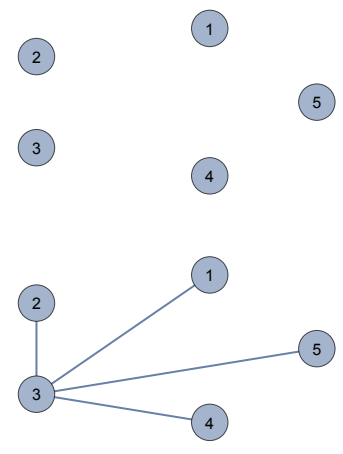

Figure 9: The network formation process is path dependent

\subsection{Relation to structural balance theory}

In this subsection we show how our main result fits with the theory of structural balance from social psychology. We first note that the absence of a link can be interpreted in our model as a self-enforcing commitment not to engage in a contest. So the absence of a negative (contest) link can be viewed as a positive link.

The theory of (strong) structural balance, first formulated by (Heider, 1946), applies to situations in which relations between agents can be either negative (antagonistic) or positive (friendship). It states that in groups of three agents, the only socially stable structures are those in which all three agents are friends (all links are positive) or two of them are friends, with the third being a common enemy (one positive and two negative links). In other words, a friendship is transitive. There are two social structures that satisfy structural balance (Cartwright and Harary, 1956): (i) all agents are friends or (ii) agents are divided into two groups, members of the same group are friends, members 
of different groups are enemies. For positive links, a network that satisfies structural balance will be a complete network or a network with two components that are cliques. For negative links, it will be either an empty network or a complete bipartite network. It has been argued in (Davis, 1967) that in many contexts we may have a situation in which all links in a triad are negative. To encompass this type of configuration, the concept of weak structural balance is proposed. The implication for the global structure when allowing for this type of triad is the emergence of additional balanced network structures. For positive links, these are networks with more than 2 components in which every component is a clique. For negative links, they are complete K-partite networks.

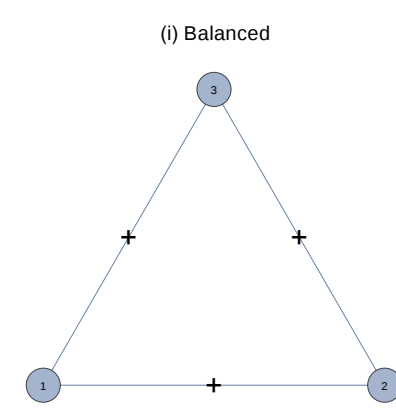

(iii) Balanced

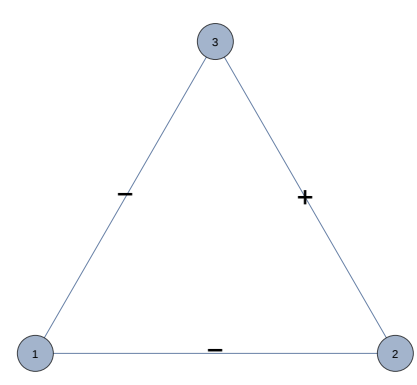

(ii) Not balanced

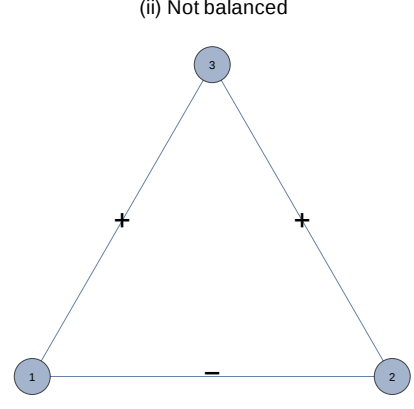

(iv) Not strongly balanced, weakly balanced

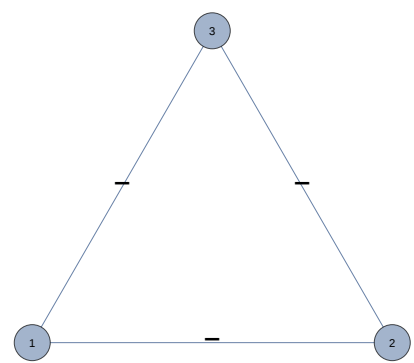

Figure 10: Structural balance

There are a number of empirical papers that find evidence in favor of structural balance theory in different settings, see for example (Antal et al., 2006, Szell et al., 2010, Sytch and Tatarynowicz, 2014). A textbook treatment of structural balance theory can be found in (Easley and Kleinberg, 2010). In our model, stable networks are always weakly balanced (they satisfy weak structural balance). Strong structural balance is satisfied in particular cases - when the stable network is a complete bipartite. It is important to note that structural balance is a concept concerning only the sign of links, which does not say anything about the intensities/weights assigned to links. Stable networks in our model are signed and weighted 4 , therefore the model yields insights that go far beyond

\footnotetext{
${ }^{14}$ We can assign the equilibrium efforts $s_{i j}^{*}, s_{j i}^{*}$ as weights to every link $g_{i j}$
} 
structural balance theory.

\section{Conclusion}

In this paper, we study a transferable contest game played on a network and explore the formation of contest networks. We provide a stylized model of contest network formation which captures the following qualitative properties of contest-type relations: (i) they are costly, (ii) the probability of a favorable outcome increases with a player's own effort and decreases with the opponent's effort, (iii) there are spillovers between contests (iv) starting a contest is a unilateral decision and ending a contest is a bilateral decision. We believe that the qualitative insights of the model are applicable to many real-world situations including competitions between divisions in companies, lobbying, litigation and armed conflict.

When the contest network is given, the model essentially leads to a definition of strength (contest centrality) in the network - a player will be strong if she does not have many opponents and if her opponents are not strong. The recursive nature of this concept of strength is a common feature of global centrality measures in networks (i.e. Katz centrality, Bonacich centrality, PageRank). However, as the contest game is a game with non-linear and non-monotone best responses we cannot hope to obtain a simple explicit representation for contest centrality. Actually, the system of equations which defines contest centrality is given with equation (11).

$$
\left(\begin{array}{cccccc}
0 & \frac{g_{12}}{\left(A_{1}+A_{2}\right)^{2}} & \frac{g_{13}}{\left(A_{1}+A_{3}\right)^{2}} & \cdots & \cdots & \frac{g_{1 n}}{\left(A_{1}+A_{n}\right)^{2}} \\
\frac{g_{21}}{\left(A_{2}+A_{1}\right)^{2}} & 0 & \frac{g_{23}}{\left(A_{2}+A_{3}\right)^{2}} & \cdots & \cdots & \frac{g_{2 n}}{\left(A_{2}+A_{n}\right)^{2}} \\
\vdots & \vdots & \ddots & & \vdots & \vdots \\
\vdots & \vdots & & \ddots & \vdots & \vdots \\
\frac{g_{n 1}}{\left(A_{n}+A_{1}\right)^{2}} & \frac{g_{n 2}}{\left(A_{n}+A_{2}\right)^{2}} & \frac{g_{n 3}}{\left(A_{n}+A_{3}\right)^{2}} & \cdot & . & 0
\end{array}\right)\left(\begin{array}{c}
A_{1} \\
A_{2} \\
\vdots \\
\vdots \\
A_{n}
\end{array}\right)=\left(\begin{array}{c}
A_{1} \\
A_{2} \\
\vdots \\
\vdots \\
A_{n}
\end{array}\right)
$$

Although, from Proposition 1, we know that system (11) has a unique solution, finding the analytical solution of (11) for an arbitrary network proved to be infeasible. However one can fairly efficiently calculate the strength of a node in the contest network using a numerical algorithm based on Proposition 8.

Even though solving for the equilibrium of the game on a given network is analytically infeasible, we show, for a general technology and cost function, that a stable network must have a very specific structure: it must be a complete $K$-partite. With more assumptions on the cost and the technology function, we are able to narrow down the set of potentially 
stable networks and provide sufficient conditions for stability. As the transferable contest is a wasteful interaction, an empty network is the efficient network structure.

There are several avenues for future research. One interesting direction would be to replace the transferable contest with a contest in which players compete for a share of an exogenous prize. This modification would not change the existence and uniqueness results from Subsection 2.2. However, in this case it is not clear why link destruction should be a bilateral decision, if the prize exists independently of the contest. A patent race (innovation contest) is a more appropriate situation to model in this way (Baye and Hoppe, 2003). This approach would extend naturally to cases where a contest is not necessarily bilateral - for instance a competition among $n$ firms on $m$ markets. 


\section{Appendix A: Proofs}

We first provide a definition of a concave game.

\section{Definition 6}

A n-player game is a concave game if (i) the strategy space (S) is given with $S=\left\{S_{1} \times\right.$ $\left.S_{2} \times \ldots \times S_{n} \mid S_{i} \subset E^{m_{i}} \wedge m_{i} \in \mathbb{N}\right\}$ and $E^{m_{i}}$ is a closed, convex and bounded subset of Euclidian spack ${ }^{15}$ and (ii) the payoff function $\pi_{i}(s)$ of every player $i$ is continuous in $\boldsymbol{s}=\left(\boldsymbol{s}_{1}, \boldsymbol{s}_{2}, \ldots, \boldsymbol{s}_{n}\right)$ and concave in $\boldsymbol{s}_{i} \in S_{i}$ for a fixed $\boldsymbol{s}_{-i} \in S_{-i}$

Proof of Proposition 1. Uniqueness: We first show that the contest game on a network is a concave game, according to Definition 6

(i) The strategy space $S$ of the game is in general unbounded, but since the transfer $R$ is finite, and the cost function $c$ is strictly increasing and convex, there exists a point $M \in \mathbb{R}$ such that $c(M)>R$. No player will wish to exert effort larger than $M$. Therefore we can bound the strategy space from above.

(ii) It is clear that the payoff function $\pi_{i}$ is continuous and twice differentiable on its domain. To show that the payoff function of player $i$ is concave in $\boldsymbol{s}_{i}$ we show that Hessian of a player's payoff function with respect that player's strategy is a negative definite matrix. To see this, note that

$$
\frac{\partial^{2} \pi_{i}}{\partial s_{i j}{ }^{2}}=\frac{\left(r+2 \phi\left(s_{j i}\right)\right)\left(\phi^{\prime \prime}\left(s_{i j}\right)\left(r+\phi\left(s_{i j}\right)+\phi\left(s_{j i}\right)\right)-2 \phi^{\prime}\left(s_{i j}\right)^{2}\right)}{\left(r+\phi\left(s_{i j}\right)+\phi\left(s_{j i}\right)\right)^{3}}-c^{\prime \prime}\left(A_{i}\right)<0
$$

The inequality in 12 holds as the first term in the difference is negative (due to the properties of function $\phi$ stated in Assumption 2) and the second term is positive (due to the strict convexity of function $c$ ). Furthermore

$$
\frac{\partial^{2} \pi_{i}}{\partial s_{i j} \partial s_{i k}}=-c^{\prime \prime}\left(A_{i}\right)<0 \forall j, k \in N_{i}
$$

Thus, the Hessian matrix $H_{i}$ of function $\pi_{i}$ with respect to $\boldsymbol{s}_{i}$ is the sum of diagonal matrix $H_{i 1}$ with diagonal elements equal to:

$$
\frac{\left(r+2 \phi\left(s_{j i}\right)\right)\left(\phi^{\prime \prime}\left(s_{i j}\right)\left(r+\phi\left(s_{i j}\right)+\phi\left(s_{j i}\right)\right)-2 \phi^{\prime}\left(s_{i j}\right)^{2}\right)}{\left(r+\phi\left(s_{i j}\right)+\phi\left(s_{j i}\right)\right)^{3}}<0
$$

and matrix $H_{i 2}$ which has all the elements equal to $-c^{\prime \prime}\left(A_{i}\right)<0 . H_{i 1}$ is a negative definite matrix and $H_{i 2}$ is a negative semidefinite matrix, thus Hessian $H_{i}=H_{i 1}+$ $H_{i 2}$ is a negative definite matrix.

\footnotetext{
$15($ Rosen, 1965) actually proves a more general result when the strategy space is coupled, that is when $S \subset E^{m}=E^{m_{1}} \times E^{m_{2}} \times \ldots \times E^{m_{n}}$ is a closed, convex and bounded set. Here we consider a special case when the strategy space is uncoupled
} 
We have proved that the contest game an a network is a concave game. Let us now define function $\sigma: S \times \mathbb{R}_{+}^{n} \rightarrow \mathbb{R}$ assigned to the contest game on a network with $\sigma(\boldsymbol{s}, \boldsymbol{z})=\sum_{i=1}^{n} z_{i} \pi_{i}(s)$. Following $($ Rosen, 1965) a concave game will have a unique Nash equilibrium if $\sigma$ is diagonally strictly concave. To prove that function $\sigma$ is diagonally strictly concave we use the result from (Goodman, 1980) which states that $\sigma(\mathbf{s}, \mathbf{z})$ will be diagonally strictly concave if the payoff functions are such that for every player $i$ : (a) $\pi_{i}(\mathbf{s})$ is strictly concave in $\mathbf{s}_{i},(\mathbf{b}) \pi_{i}(\mathbf{s})$ is convex in $\mathbf{s}_{-i}$ and $(\mathbf{c}) \sigma(\mathbf{s}, \mathbf{z})$ is concave in $\mathbf{s}$ for some $\mathbf{z} \geq 0$. We have already shown (a). To see that (b) holds, note that (when $g_{i j}=1$ )

$$
\frac{\partial^{2} \pi_{i}}{\partial s_{j i}^{2}}=\frac{\left(r+2 \phi\left(s_{i j}\right)\right)\left(2 \phi^{\prime}\left(s_{j i}\right)^{2}-\phi^{\prime \prime}\left(s_{j i}\right)\left(r+\phi\left(s_{i j}\right)+\phi\left(s_{j i}\right)\right)\right)}{\left(r+\phi\left(s_{i j}\right)+\phi\left(s_{j i}\right)\right)^{3}}>0
$$

Furthermore, $\left(\forall g_{j k} \in g: k \neq i\right), \frac{\partial^{2} \pi_{i}}{\partial s_{j k}^{2}}=0$ and $\frac{\partial^{2} \pi_{i}}{\partial s_{j k} \partial s_{l t}}=0$ for any other combination of players $j, k, l$ and $t$. Thus, the Hessian of $\pi_{i}$ with respect to $s_{-i}$ is a diagonal matrix with all entries positive or zero and therefore positive semi-definite.

To prove (c) choose $\boldsymbol{z}=\mathbf{1}$. Then:

$$
\sigma(\mathbf{s}, \mathbf{1})=\sum_{i=1}^{n} \sum_{j \in N_{i}}\left(\frac{\phi\left(s_{i j}\right)}{\phi\left(s_{i j}\right)+\phi\left(s_{j i}\right)+r}-\frac{\phi\left(s_{j i}\right)}{\phi\left(s_{i j}\right)+\phi\left(s_{j i}\right)+r}-c\left(A_{i}\right)\right)=-\sum_{i=1}^{n} c\left(A_{i}\right)
$$

The last equality above holds since in the first sum above $\frac{\phi\left(s_{i j}\right)}{\phi\left(s_{i j}\right)+\phi\left(s_{j i}\right)+r}$ appears exactly once with a positive sign (as a part of payoff function $\pi_{i}$ ) and exactly once with a negative sign (as a part of function $\pi_{j}$ ). Function $-\sum_{i} c\left(A_{i}\right)$ is strictly concave due to the strict convexity of the cost function $c$. Hence, (c) also holds. We have shown that there is a unique equilibrium of the game on a fixed network.

Interiority: Consider two players $i$ and $j$ such that $g_{i j}=1$. Let us first prove that it cannot be $s_{i j}^{*}=s_{j i}^{*}=0 \forall r>0$. Assume this is not true. Then, the payoff of both players in contest $g_{i j}$ will be 0 . Consider now the deviation of player $i$ from $s_{i j}^{*}=0$ to $s_{i j}=r$. The probability of winning for player $i$ becomes $p_{i j}=\frac{\phi(r)}{\phi(r)+r}=\alpha>0$ and the probability of losing $p_{j i}$ is still 0 . This deviation is profitable as long as $c\left(A_{i}^{*}+r\right)-c\left(A_{i}^{*}\right)<\alpha$. As $c$ is continuous, we can always find $r$ such that $\left|c\left(A_{i}^{*}+r\right)-c\left(A_{i}^{*}\right)\right|<\alpha$. Therefore, in this case there always exists $r$ such that the deviation from $s_{i j}=0$ to $s_{i j}=r$ is profitable. For such $r$ it cannot be that $s_{i j}^{*}=s_{j i}^{*}=0$ in the equilibrium.

Let us now prove that for two connected players $i$ and $j$ it cannot be that $s_{i j}^{*} \neq 0$ $\wedge s_{j i}^{*}=0 \forall r>0$. Suppose that, contrary to what is asserted, this is the case for some two players $i$ and $j$. That is, suppose $s_{i j}^{*} \neq 0$ and $s_{j i}^{*}=0$. Equation (4) implies that:

$$
\left.\frac{\partial \pi_{i}}{\partial s_{i j}}\right|_{\left(s_{i j}^{*}, s_{j i}^{*}\right)}=\frac{(r+2 \phi(0)) \phi^{\prime}\left(s_{i j}^{*}\right)}{\left(r+\phi\left(s_{i j}^{*}\right)+\phi(0)\right)^{2}}-c^{\prime}\left(A_{i}^{*}\right)=\frac{r \phi^{\prime}\left(s_{i j}^{*}\right)}{\left(r+\phi\left(s_{i j}^{*}\right)\right)^{2}}-c^{\prime}\left(A_{i}^{*}\right)=0
$$


But we can always find $r$ small enough such that (13) cannot hold for any $s_{i j}^{*}>0$ and $A_{i}^{*}>0$. Indeed, since $R$ is finite and the number of nodes in the network is finite, then $A_{i}^{*}$ must be finite for every $i$. For any cost function $c \in C^{2}$ which satisfies Assumption 1 we can find $U>0$ such that $c^{\prime}(x)<U$ for every finite $x$. Thus $c^{\prime}\left(A_{i}^{*}\right)$ is bounded from above. Furthermore, we can always choose $r>0$ small enough such that $\frac{r \phi^{\prime}\left(s_{i j}^{*}\right)}{\left(r+\phi\left(s_{i j}^{*}\right)\right)^{2}}>U$ $\forall s_{i j}^{*} \in[0, M]$, since for any $s_{i j}^{*}>0$ we have that $\frac{r \phi^{\prime}\left(s_{i j}^{*}\right)}{\left(r+\phi\left(s_{i j}^{*}\right)\right)^{2}} \rightarrow \infty$ when $r \rightarrow 0$.

Proof of proposition 2. Expressing $\phi\left(s_{i j}^{*}\right)$ from (4) we get that in the equilibrium:

$$
\phi\left(s_{i j}^{*}\right)=\frac{2\left[\phi^{\prime}\left(s_{i j}^{*}\right)\right]^{2} c^{\prime}\left(A_{j}^{*}\right) \phi^{\prime}\left(s_{j i}^{*}\right)}{\left(\phi^{\prime}\left(s_{i j}^{*}\right) c^{\prime}\left(A_{j}^{*}\right)+\phi^{\prime}\left(s_{j i}^{*}\right) c^{\prime}\left(A_{i}^{*}\right)\right)^{2}}-\frac{r}{2}
$$

Let us define function $f$ with:

$$
f(x, y ; a, b, r)=\frac{2 a^{2} c^{\prime}(y) b}{\left(a c^{\prime}(y)+b c^{\prime}(x)\right)^{2}}-\frac{r}{2}
$$

We first notice that 16 holds.

$$
\begin{aligned}
& \frac{\partial f}{\partial x}=-\frac{4 a^{2} b^{2} c^{\prime}(y)}{\left(b c^{\prime}(x)+a c^{\prime}(y)\right)^{3}} \\
& \frac{\partial f}{\partial y}=\frac{2 a^{2} b\left(b c^{\prime}(x)-a c^{\prime}(y)\right)}{\left(b c^{\prime}(x)+a c^{\prime}(y)\right)^{3}}
\end{aligned}
$$

When $0<a \leq b$ the signs of derivatives in (16) are: $\frac{\partial f}{\partial x}<0$ and $\frac{\partial f}{\partial y} \lessgtr 0$ when $x \lessgtr y$. Recall also that from (5) $A_{j}^{*}>A_{i}^{*} \Rightarrow s_{i j}^{*}>s_{j i}^{*}$. Furthermore, since $\phi$ is increasing and concave, $s_{i j}^{*}>s_{j i}^{*} \Rightarrow \phi^{\prime}\left(s_{i j}^{*}\right) \leq \phi^{\prime}\left(s_{j i}^{*}\right)$. Now, setting $a=\phi^{\prime}\left(s_{i j}^{*}\right)$ and $b=\phi^{\prime}\left(s_{j i}^{*}\right)$ in 16 it directly follows that $A_{c}^{*}>A_{b}^{*}>A_{a}^{*}$ implies $s_{a b}^{*}>s_{a c}^{*}$ and $s_{b a}^{*}>s_{c a}^{*}$. To prove that $\pi_{i}\left(g_{a c}\right)>\pi_{i}\left(g_{a b}\right)$ we plug (14) in (8). After some algebra we get:

$$
\pi_{i}\left(g_{i j}\right)=1-\frac{2 \phi^{\prime}\left(s_{j i}^{*}\right) c^{\prime}\left(A_{i}^{*}\right)}{\phi^{\prime}\left(s_{i j}^{*}\right) c^{\prime}\left(A_{j}^{*}\right)+\phi^{\prime}\left(s_{j i}^{*}\right) c^{\prime}\left(A_{i}^{*}\right)}-\left(c\left(A_{i}^{*}\right)-c\left(A_{i}^{*}-s_{i j}^{*}\right)\right)
$$

It is clear that $\pi_{i}\left(g_{i j}\right)$ is strictly increasing in $A_{j}^{*}$. Thus, $A_{c}^{*}>A_{b}^{*}>A_{a}^{*} \Rightarrow \pi_{i}\left(g_{a c}\right)>$ $\pi_{i}\left(g_{a b}\right)$

Proof of Lemma 1. Let us write (14) for contest $g_{a b}$ as:

$$
\phi\left(s_{a b}^{*}\right)=\frac{2\left[\phi^{\prime}\left(s_{a b}^{*}\right)\right]^{2} c^{\prime}\left(A_{b}^{*}-s_{b a}^{*}+s_{b a}^{*}\right) \phi^{\prime}\left(s_{b a}^{*}\right)}{\left(\phi^{\prime}\left(s_{a b}^{*}\right) c^{\prime}\left(A_{b}^{*}-s_{b a}^{*}+s_{b a}^{*}\right)+\phi^{\prime}\left(s_{b a}^{*}\right) c^{\prime}\left(A_{a}^{*}-s_{a b}^{*}+s_{a b}^{*}\right)\right)^{2}}-\frac{r}{2}
$$

As a player assumes that actions in all other contests will remain fixed when she creates a new link, $s_{a c}^{*}$ and $s_{c a}^{*}$ are determined as the solution of system 17) (see Example 1 for 
details on the creation of a new link in the network):

$$
\begin{aligned}
\phi\left(s_{a c}^{*}\right) & =\frac{2\left[\phi^{\prime}\left(s_{a c}^{*}\right)\right]^{2} c^{\prime}\left(A_{c}^{*}+s_{c a}^{*}\right) \phi^{\prime}\left(s_{c a}^{*}\right)}{\left(\phi^{\prime}\left(s_{a c}^{*}\right) c^{\prime}\left(A_{c}^{*}+s_{c a}^{*}\right)+\phi^{\prime}\left(s_{c a}^{*}\right) c^{\prime}\left(A_{a}^{*}-s_{c a}^{*}+s_{a c}^{*}\right)\right)^{2}}-\frac{r}{2} \\
\phi\left(s_{c a}^{*}\right) & =\frac{2\left[\phi^{\prime}\left(s_{c a}^{*}\right)\right]^{2} c^{\prime}\left(A_{a}^{*}+s_{a c}^{*}\right) \phi^{\prime}\left(s_{a c}^{*}\right)}{\left(\phi^{\prime}\left(s_{c a}^{*}\right) c^{\prime}\left(A_{a}^{*}+s_{a c}^{*}\right)+\phi^{\prime}\left(s_{a c}^{*}\right) c^{\prime}\left(A_{c}^{*}-s_{a c}^{*}+s_{c a}^{*}\right)\right)^{2}}-\frac{r}{2}
\end{aligned}
$$

It is clear that, $A_{c}^{*}+s_{c a}^{*}>A_{c}^{*} \geq A_{b}^{*}$. Since $A_{c}^{*}+s_{c a}^{*}>A_{b}^{*}$ Proposition 2 implies that this deviation is profitable.

Proof of Corollary 1: Suppose that the claim of the corollary is not true. If link $g_{a b}$ is not profitable for player $a$ then it is not profitable for player $b$, as $a$ is stronger than $b$. Then link $g_{a b}$ cannot be a part of a stable network. Therefore, it must be that link $g_{a b}$ is profitable for player $a$. Let $c \in \mathscr{A}_{j+k} k \geq 0$ be a node such that link $g_{a c}$ does not exist. Then, from Lemma 1 the deviation of player $a$ in which she destroys link $g_{a b}$ and creates link $g_{a c}$ is profitable.

Proof of Lemma 2: We use a proof by contradiction. Assume that the claim of the lemma does not hold. Then there are at least two components in a stable network. Choose two components $\left(C_{1}\right.$ and $\left.C_{2}\right)$ from the network such that the weakest player in the network $\left(w_{1}\right)$ belongs to $C_{1}$. All opponents of $w_{1}$ must find the contest with $w_{1}$ beneficial. Otherwise the network would not be stable. Then, the strongest player in $C_{2}$ (denote her with $a_{2}$ ) by Lemma 1 has an incentive to form a link with $w_{1}$ instead of link with one of her current opponents which are not weaker than $w_{1}$. If $\left|C_{2}\right|=1$ then $a_{2}$ has an incentive to form a link with $w_{1}$ as she is stronger than the strongest member of $C_{1}$ (since $A_{a_{2}}^{*}=0$ in this case).

Proof of Lemma 3: Consider two nodes $a, b \in \mathscr{A}$. Let us first prove that they must have the same degree. Suppose that this is not true, that is, suppose that in a stable network $d_{b}>d_{a}$. It cannot be that $N_{a} \subset N_{b}$ because then the total spending of $a$ and $b$ would not be equal (they would not belong to the same class). As $N_{a} \neq N_{b}$, there must exist nodes $h \in N_{a} \backslash N_{b}$ and $k \in N_{b} \backslash N_{a}$. Suppose that, without loss of generality, $A_{k}^{*} \geq A_{h}^{*}$. Then it would be beneficial for player $a$ to replace link $g_{a h}$ with link $g_{a k}$ according to Lemma 1. This is in contradiction with the assumption that the network is stable. So it must be $d_{a}=d_{b}$.

Let us now prove that $N_{a}=N_{b}$. Again, assume this is not the case. Then we can find two nodes $h \in N_{a} \backslash N_{b}$ and $k \in N_{b} \backslash N_{a}$ such that, without loss of generality, $A_{k}^{*} \geq A_{h}^{*}$. But then it would be better for player $a$ to replace link $g_{a h}$ with link $g_{a k}$ according to Lemma 1. Thus, network $g$ cannot be stable. The assumption that $N_{a} \neq N_{b}$ led us to the contradiction and must be rejected. 
Proof of Lemma 4: We again use a proof by contradiction. Suppose there are two different classes of attackers and denote them with $\mathscr{A}_{1}$ and $\mathscr{A}_{2}$ and let $A_{2}^{*}>A_{1}^{*}$. As Lemma 3 implies that all members of the same class of attackers have the same neighborhood, we restrict our attention to representative nodes $a \in \mathscr{A}_{1}$ and $b \in \mathscr{A}_{2}$.

Let us first prove that it must be $\pi_{a}=\pi_{b}$. Assume this is not the case. Then it must be that $N_{a} \neq N_{b}$. Since $A_{2}^{*}>A_{1}^{*}$ there are two possible situations that we need to consider.

(i) $N_{a} \subset N_{b}$. If $\pi_{a}>\pi_{b}$ player $b$ would prefer to imitate $a$ 's linking strategy (this is feasible as she is an attacker), and if $\pi_{b}>\pi_{a}$ then $a$ will have an incentive to mimic $b^{16}$.

(ii) $N_{a} \not \subset N_{b} \Longrightarrow\left(\exists k \in N_{a} \backslash N_{b} \wedge \exists h \in N_{b} \backslash N_{a}\right)$. In this case, if $A_{k}^{*} \geq A_{h}^{*}$ Lemma 1 implies that $b$ has a profitable deviation. If $A_{k}^{*}<A_{h}^{*}$ the same Lemma implies that $a$ has a profitable deviation. Hence, in a stable network it must be $\pi_{a}=\pi_{b}$.

We now prove that in a stable network it must be that $N_{a}=N_{b}$. As $A_{2}^{*}>A_{1}^{*}$ it must be that $d_{b}>d_{a}$ or the distributions of total spending of $a^{\prime} s$ and $b^{\prime} s$ opponents are different. We show that in both cases there exists a feasible deviation which makes one of the players better off.

Let us first consider the case when $d_{b}>d_{a}$. If $N_{a} \subset N_{b}$ we have (i) from above. If $N_{a} \not \subset N_{b}$ there must exist nodes $k \in N_{a} \backslash N_{b}$ and $h \in N_{b} \backslash N_{a}$. If $A_{k}^{*} \geq A_{h}^{*}$ then player $b$ would be better off by replacing contest $g_{b h}$ with $g_{b k}$ by Lemma 1 . If $A_{k}^{*}<A_{h}^{*}$ player $a$ can make an analogous profitable deviation.

If $d_{a}=d_{b}$ then, since $A_{2}^{*}>A_{1}^{*}$ the strengths of $a^{\prime} s$ opponents are different than the strengths of $b^{\prime} s$ opponents. Let $q$ be the strongest node from $\left(N_{a} \cup N_{b}\right) \backslash\left(N_{a} \cap N_{b}\right) \neq \emptyset$. If link $g_{a q}$ exists, then it is profitable for $a$ to replace $g_{a q}$ with $g_{a y}$ where $y \in N_{b} \backslash N_{a}$. If $g_{b q}$ exists, then the profitable deviation is switching from $g_{b q}$ to $g_{b z}$ where $z \in N_{a} \backslash N_{b}$.

Proof of Lemma 5: If there are only two classes of nodes in a stable network $\left(\mathscr{A}_{1}\right.$ and $\left.\mathscr{A}_{2}\right)$ then there are no mixed types. Suppose there are more than two classes of nodes in a stable network. First, let us consider the strongest mixed type class $\left(\mathscr{A}_{2}\right)$. A node $m \in \mathscr{A}_{2}$ must be connected to all nodes from $\mathscr{A}_{1}$. This is because a mixed type player $m$ must be connected with at least one stronger player, which must be an attacker because of the choice of $m$. Then, Lemma 4 implies that $m$ must be connected to all players from class $\mathscr{A}_{1}$.

Let us now prove that all members of the class $\mathscr{A}_{2}$ have the same neighborhood. Suppose this is not true. Let $\left\{m_{1}, m_{2}\right\} \subset \mathscr{A}_{2} \wedge N_{m_{1}} \neq N_{m_{2}}$. As $\left(\mathscr{A}_{1} \subset N_{m_{1}} \wedge \mathscr{A}_{1} \subset\right.$ $\left.N_{m_{2}}\right) \Longrightarrow\left(\left(N_{m_{1}} / N_{m_{2}}\right) \cup\left(N_{m_{2}} / N_{m_{1}}\right)\right) \cap \mathscr{A}_{1}=\emptyset$. Thus, if $N_{m_{1}}$ and $N_{m_{2}}$ differ, they must differ only in the part where $m_{1}$ and $m_{2}$ have control over their links. It cannot

\footnotetext{
${ }^{16}$ Recall that we assume that when a player is indifferent between two actions he prefers to have less links.
} 
be $N_{m_{1}} \subset N_{m_{2}} \vee N_{m_{2}} \subset N_{m_{1}}$ because then it would be $A_{m_{1}}^{*} \neq A_{m_{2}}^{*}$. Consider two nodes, $k \in N_{m_{1}} \backslash N_{m_{2}}$ and $l \in N_{m_{2}} \backslash N_{m_{1}}$. Note that sets $N_{m_{1}} \backslash N_{m_{2}}$ and $N_{m_{2}} \backslash N_{m_{1}}$ cannot be empty. If $A_{k}^{*} \geq A_{l}^{*}$ then $m_{2}$ has a profitable deviation (switching from $g_{m_{2} l}$ to $g_{m_{2} k}$ ). If $A_{k}^{*}<A_{l}^{*}$, then $m_{1}$ has an analogous profitable deviation.

Let $\mathscr{A}_{3}$ be the third strongest class in the network. If $K=3$ then, by definition, all players $\mathscr{A}_{2}$ must be connected to some players of $\mathscr{A}_{3}$, because otherwise they would not be mixed types. Note that if player $i \in \mathscr{A}_{3}$ is connected to some player from class $\mathscr{A}_{2}$ that she is connected to all players from class $\mathscr{A}_{2}$ since we have shown that all members of class $\mathscr{A}_{2}$ have the same neighborhood. If there exists some player $j \in A_{3}$ who is not connected to all players from $\mathscr{A}_{2}$ then she is only connected to all players from $\mathscr{A}_{1}$ but then $i$ and $j$ cannot belong to the same class. So, for $K=3$ the claim of the lemma holds.

If $K>3$, then $\mathscr{A}_{3}$ is a class of mixed types. Corollary 1 implies that all members of $\mathscr{A}_{1}$ must be connected to all members of $\mathscr{A}_{3}$ since they are connected to all members of $\mathscr{A}_{2}$ and $A_{2}^{*}<A_{3}^{*}$.

We now show that all players from $\mathscr{A}_{2}$ are connected to all players from $\mathscr{A}_{3}$. Suppose that there exist players $i \in \mathscr{A}_{2}$ and $j \in \mathscr{A}_{3}$ such that $g_{i j}=0$. If this is the case we show that there will always exist a profitable deviation some player in the network.

Since all players from $\mathscr{A}_{2}$ have the same neighborhood then there will be no links between members of class $\mathscr{A}_{2}$ and $\mathscr{A}_{3}$. This means that players from $\mathscr{A}_{3}$ lose only in contests with players from $\mathscr{A}_{1}$. Because of this, they have control over all of their links except links that connect them to players in $\mathscr{A}_{1}$. Furthermore, $A_{2}^{*}<A_{3}^{*} \Longrightarrow N_{i} \neq N_{j}$.

Suppose first that $\pi_{i} \neq \pi_{j} i \in \mathscr{A}_{2}, j \in \mathscr{A}_{3}$.

(i) $N_{i} \subset N_{j}$ then $j$ can destroy links towards all players in $N_{j} / N_{i}$ and increase her payoff $i$ (if $\pi_{i}>\pi_{j}$ ), or player $i$ can create links to all players in $N_{j} / N_{i}$ and increase her payoff (if $\pi_{i}<\pi_{j}$ )

(ii) $N_{i} \not \subset N_{j} \Longrightarrow\left(\exists k \in N_{i} \backslash N_{j} \wedge \exists h \in N_{j} \backslash N_{h}\right)$. But then, if $A_{k}^{*} \geq A_{h}^{*}$ Lemma 1 implies that $j$ has a profitable deviation, and if $A_{k}^{*}<A_{h}^{*}$, the same lemma implies that $i$ has a profitable deviation.

If $\pi_{i}=\pi_{j}$ since $A_{3}^{*}>A_{2}^{*}$ then it must be that $d_{j}>d_{i}$ or that the distributions of total spending of $i^{\prime} s$ and $j^{\prime} s$ opponents are different. We show that in both cases there exists a profitable deviation.

Let us first consider the case when $d_{i}>d_{j}$. If $N_{i} \subset N_{j}$ we have (i) from above. If $N_{i} \not \subset N_{j}$ we have an analogue of (ii) from above.

If $d_{i}=d_{j}$ then, since $A_{3}^{*}>A_{2}^{*}$, the strengths (total equilibrium spending) of $i^{\prime} s$ opponents are different than the strengths of $j^{\prime} s$ opponents. Let $q$ be the strongest node from $\left(N_{a} \cup N_{b}\right) \backslash\left(N_{a} \cap N_{b}\right) \neq \emptyset$. If link $g_{i q}$ exists, then it is profitable for $i$ to switch from $q$ 
to any node in the set $N_{j} \backslash N_{i}$. If $g_{j q}$ exists, then the profitable deviation for $b$ is switching from $q$ to some node in $N_{i} \backslash N_{j}$.

Thus, we have shown that in a stable network it cannot happen that there are no links between members of $\mathscr{A}_{2}$ and $\mathscr{A}_{3}$.

Using the same technique as above, we can show that all players from $\mathscr{A}_{k}$ must be connected to all players from $\mathscr{A}_{k+1}$. Since the number of nodes in the network is finite, the number of classes is finite and this procedure reaches $\mathscr{A}_{K}$ in a finite number of steps.

Proof of Proposition 4: Suppose that the claim does not hold, so let $A_{k}^{*}<A_{k+1}^{*}$ and assume that $\left|\mathscr{A}_{k}\right|<\left|\mathscr{A}_{k+1}\right|$. Note that (4) implies that $s_{i j}^{*}=s_{i h}^{*} \forall\{i, j, h\} \in N \wedge\{j, h\} \in$ $\mathscr{A}_{l}, l \in\{1,2, \ldots, K\}$. So, in a stable network, for any two players $x, y$ such that $x \in \mathscr{A}_{k}$ and $y \in \mathscr{A}_{k+1}$, we have that $A_{k}^{*}=\sum_{i \neq k}\left|\mathscr{A}_{i}\right| s_{x i}^{*}$ and $A_{k+1}^{*}=\sum_{i \neq k+1}\left|\mathscr{A}_{i}\right| s_{y i}^{*}$. Recall that due to strict convexity of the cost function, $A_{a}^{*}>A_{b}^{*} \Longrightarrow s_{a j}^{*}<s_{b j}^{*} \forall\left(j \in N: g_{a j}=g_{b j}=1\right)$. This implies that $s_{x j}^{*}>s_{y j}^{*} \forall j \in\left\{\mathscr{A}_{1}, \mathscr{A}_{2} . ., \mathscr{A}_{K}\right\} \backslash\left\{\mathscr{A}_{k}, \mathscr{A}_{k+1}\right\}$. Furthermore, since $A_{k}^{*}<A_{k+1}^{*}$ we have that $s_{x y}^{*}>s_{y x}^{*}$. But then $\left|\mathscr{A}_{k}\right|<\left|\mathscr{A}_{k+1}\right| \Rightarrow \sum_{i \neq k}\left|\mathscr{A}_{i}\right| s_{x i}>\sum_{i \neq k+1}\left|\mathscr{A}_{i}\right| s_{y i} \Longrightarrow A_{k}^{*}>$ $A_{k+1}^{*}$. This is in contradiction with the fact that $A_{k}^{*}<A_{k+1}^{*}$. The assumption $A_{k}^{*}<A_{k+1}^{*}$ lead us to contradiction so it must be rejected.

Proof of Proposition 5: In a complete bipartite network no player has an incentive to form a link, as the only links that can be formed are links with players who have the same total equilibrium spending $A^{*}$. Let us show that no player will wish to sever a link if the condition from the statement of the proposition holds. Suppose that $i \in \mathscr{K}_{1}$ and $j \in \mathscr{K}_{2}$ and $\left|\mathscr{K}_{1}\right|>\left|\mathscr{K}_{2}\right|$. Player $j \in \mathscr{K}_{2}$ will always wish to sever link with $i \in \mathscr{K}$, as $i$ is stronger than $j$. From (5) we have that a player $i \in \mathscr{K}_{1}$ will wish to keep link $g_{i j}$ when

$$
\frac{\phi^{\prime}\left(s_{i j}^{*}\right) c^{\prime}\left(A_{j}^{*}\right)-\phi^{\prime}\left(s_{j i}^{*}\right) c^{\prime}\left(A_{i}^{*}\right)}{\phi^{\prime}\left(s_{i j}^{*}\right) c^{\prime}\left(A_{j}^{*}\right)+\phi^{\prime}\left(s_{j i}^{*}\right) c^{\prime}\left(A_{i}^{*}\right)}-c\left(A_{i}^{*}\right)+c\left(A_{i}^{*}-s_{i j}^{*}\right)>0
$$

where

$$
s_{i j}^{*}=\phi^{-1}\left(\frac{2\left[\phi^{\prime}\left(s_{i j}^{*}\right)\right]^{2} c^{\prime}\left(A_{j}^{*}\right) \phi^{\prime}\left(s_{j i}^{*}\right)}{\left(\phi^{\prime}\left(s_{i j}^{*}\right) c^{\prime}\left(A_{j}^{*}\right)+\phi^{\prime}\left(s_{j i}^{*}\right) c^{\prime}\left(A_{i}^{*}\right)\right)^{2}}-\frac{r}{2}\right)
$$

when $\phi$ is identity mapping $(18)$ is reduced to:

$$
\frac{c^{\prime}\left(A_{j}^{*}\right)-c^{\prime}\left(A_{i}^{*}\right)}{c^{\prime}\left(A_{j}^{*}\right)+c^{\prime}\left(A_{i}^{*}\right)}>c\left(A_{i}^{*}\right)-c\left(A_{i}^{*}-\frac{2 c^{\prime}\left(A_{j}^{*}\right)}{c^{\prime}\left(A_{i}^{*}\right)+c^{\prime}\left(A_{j}^{*}\right)}-\frac{r}{2}\right)
$$

Proof of Proposition 6. Setting $\phi$ to be the identity mapping, $c(x)=x^{2}$ and letting 
$r \rightarrow 0$ from (4) we get:

$$
s_{i j}^{*}=\sqrt{\frac{k_{j}}{\sqrt{k_{i} k_{j}}\left(\sqrt{k_{i}}+\sqrt{k_{j}}\right)^{2}}}
$$

where $i \in \mathscr{K}_{1}$ and $j \in \mathscr{K}_{2}$. To prove the claim it is enough to find conditions under which $i$ prefers not to destroy a link $g_{i j}$. Due to convexity of the cost function, if player $i$ does not wish to sever a single link, she will not want to sever multiple links. The net payoff from a link $g_{i j}$ for player $i$ defined in (8) becomes (20).

$$
\pi_{i}\left(s_{i j}^{*}, s_{j i}^{*}\right)=\frac{s_{i j}^{*}-s_{j i}^{*}}{s_{i j}^{*}+s_{j i}^{*}}-\left(k_{2} s_{i j}^{*}\right)^{2}+\left(\left(k_{2}-1\right) s_{i j}^{*}\right)^{2}
$$

Plugging (19) into 20) we get:

$$
\pi_{i}\left(g_{i j}\right)=\frac{\sqrt{k_{1}}-\sqrt{k_{2}}}{\sqrt{k_{1}}+\sqrt{k_{2}}}-\frac{k_{1}\left(2 k_{2}-1\right)}{\sqrt{k_{1} k_{2}}\left(2 \sqrt{k_{1} k_{2}}+k_{1}+k_{2}\right)}
$$

and after some manipulation we have that:

$$
\pi_{i}\left(g_{i j}\right)>0 \Leftrightarrow k_{1}^{\frac{3}{2}}+k_{1}\left(1+k_{1}\right) \sqrt{k_{2}}-k_{1}^{\frac{3}{2}} k_{2}-3 k_{1} k_{2}^{\frac{3}{2}}-\sqrt{k_{1}} k_{2}^{2}>0
$$

which finally gives:

$$
\pi_{i}\left(g_{i j}\right)>0 \Leftrightarrow k_{1}>\frac{1-4 k_{2}+6 k_{2}^{2}+\left(2 k_{2}-1\right) \sqrt{1-4 k_{2}+8 k_{2}^{2}}}{2 k_{2}}
$$

Proof of Proposition 8: It has been proved in Goodman, 1980) that $G+G^{\prime}$ is negative definite if function $\sigma: S \times \mathbb{R}_{+}^{n} \rightarrow \mathbb{R}$ defined in the proof of Proposition 1 satisfies conditions (a), (b), (c) from that proof. We have proved in the proof of Proposition 1 that this is true.

\section{Appendix B: An Alternative Formulation}

Suppose that, instead of the contest game with a general convex cost function, we consider a Colonel Blotto game with the contest success function (2). That is, suppose that each player is endowed with an equal amount of resources (time) and the strategy is to distribute the resources across different contests. Define the budget constraint by: $\sum_{j \in N_{i}} s_{i j}=1 \forall i, j$. Following (Rosen, 1965) we can easily show that Proposition 1 will still hold.

Let $\lambda_{i}$ denote the Lagrange multiplier associated with the budget constraint for agent 
$i$. The first-order conditions that characterize behavior in contest $g_{i j}$ are given by:

$$
\begin{gathered}
\frac{\left(r+2 \phi\left(s_{j i}^{*}\right)\right) \phi^{\prime}\left(s_{i j}^{*}\right)}{\left(r+\phi\left(s_{i j}^{*}\right)+\phi\left(s_{j i}^{*}\right)\right)^{2}}-\lambda_{i}=0 \\
\frac{\left(r+2 \phi\left(s_{i j}^{*}\right)\right) \phi^{\prime}\left(s_{j i}\right)^{*}}{\left(r+\phi\left(s_{i j}^{*}\right)+\phi\left(s_{j i}^{*}\right)\right)^{2}}-\lambda_{j}=0 \\
\sum_{k \in N_{i}} s_{i k}^{*}=1, \sum_{k \in N_{j}} s_{j k}^{*}=1
\end{gathered}
$$

which gives:

$$
\frac{\left(r+2 \phi\left(s_{j i}^{*}\right)\right) \phi^{\prime}\left(s_{i j}^{*}\right)}{\left(r+2 \phi\left(s_{i j}^{*}\right)\right) \phi^{\prime}\left(s_{j i}^{*}\right)}=\frac{\lambda_{i}}{\lambda_{j}}
$$

Thus, the role of $\lambda_{i}$ is analogous to the role of $A_{i}^{*}$. Higher $A_{i}^{*}$ implies a higher marginal cost of effort, and $\lambda_{i}$ is the shadow price of resources for player $i$.

\section{References}

T. Antal, P. L. Krapivsky, and S. Redner. Social balance on networks: The dynamics of friendship and enmity. Physica D: Nonlinear Phenomena, 224(1):130-136, 2006.

M. R. Baye and H. C. Hoppe. The strategic equivalence of rent-seeking, innovation, and patent-race games. Games and Economic Behavior, 44(2):217-226, 2003.

D. Cartwright and F. Harary. Structural balance: a generalization of heider's theory. Psychological review, 63(5):277, 1956.

J. A. Davis. Clustering and structural balance in graphs. Human relations, 1967.

D. Easley and J. Kleinberg. Networks, Crowds, and Markets: Reasoning About a Highly Connected World. Cambridge University Press, New York, NY, USA, 2010.

J. Franke and T. Ozturk. Conflict networks. Journal of Public Economics, 126:104 - 113, 2015.

J. C. Goodman. Note on existence and uniqueness of equilibrium points for concave n-person games. Econometrica, 48(1), 1980.

S. Goyal and F. Vega-Redondo. Structural holes in social networks. Journal of Economic Theory, 137(1):460-492, 2007.

S. Goyal, A. Vigier, and M. Dziubinski. Conflict and networks. In The Oxford Handbook of the Economics of Networks. 2016. 
F. Heider. Attitudes and cognitive organization. The Journal of psychology, 21(1):107$112,1946$.

T. Hiller. Friends and enemies: A model of signed network formation. Available at SSRN $2371249,2013$.

A. L. Hillman and J. G. Riley. Politically contestable rents and transfers*. Economics \&6 Politics, 1(1):17-39, 1989.

R. Inderst, H. M. Müller, and K. Wärneryd. Distributional conflict in organizations. European Economic Review, 51(2):385-402, 2007.

M. O. Jackson. Social and economic networks. Princeton university press Princeton, 2008.

M. O. Jackson and S. Nei. Networks of military alliances, wars, and international trade. Proceedings of the National Academy of Sciences, 112(50):15277-15284, 2015.

M. O. Jackson and A. Watts. On the formation of interaction networks in social coordination games. Games and Economic Behavior, 41(2):265-291, 2002.

H. Jia, S. Skaperdas, and S. Vaidya. Contest functions: Theoretical foundations and issues in estimation. International Journal of Industrial Organization, 31(3):211-222, 2013.

M. König, D. Rohner, M. Thoenig, and F. Zilibotti. Networks in conflict: Theory and evidence from the great war of africa. University of Zurich, UBS International Center of Economics in Society, Working Paper, (14), 2015.

A. O. Krueger. The political economy of the rent-seeking society. The American economic review, 64(3):291-303, 1974.

J. S. Levy and W. R. Thompson. Causes of war. John Wiley \& Sons, 2011.

K. O. Nti. Comparative statics of contests and rent-seeking games. International Economic Review, pages 43-59, 1997.

J. Pfeffer and W. L. Moore. Power in university budgeting: A replication and extension. Administrative Science Quarterly, pages 637-653, 1980.

J. B. Rosen. Existence and uniqueness of equilibrium points for concave n-person games. Econometrica: Journal of the Econometric Society, pages 520-534, 1965. 
M. Sytch and A. Tatarynowicz. Friends and foes: The dynamics of dual social structures. Academy of Management Journal, 57(2):585-613, 2014.

M. Szell, R. Lambiotte, and S. Thurner. Multirelational organization of large-scale social networks in an online world. Proceedings of the National Academy of Sciences, 107(31): 13636-13641, 2010.

S. Szymanski. The economic design of sporting contests. Journal of economic literature, 41(4):1137-1187, 2003.

G. Tullock. The welfare costs of tariffs, monopolies, and theft. Economic Inquiry, 5(3): 224-232, 1967.

A. Watts. A dynamic model of network formation. Games and Economic Behavior, 34: 331341, 2001. 